\title{
THE VISIBLE IMAGING SYSTEM (VIS) FOR THE POLAR
}

L. A. FRANK, J. B. SIGWARTH, J. D. CRAVEN, J. P. CRAVENS, J. S. DOLAN, M. R. DVORSKY, P. K. HARDEBECK, J. D. HARVEY and D. W. MULLER

Department of Physics and Astronomy, The University of Iowa, lowa City, IA 52242, U.S.A.

(Received 26 July, 1993)

\begin{abstract}
The Visible Imaging System (VIS) is a set of three low-light-level cameras to be flown on the POLAR spacecraft of the Global Geospace Science (GGS) program which is an element of the International Solar-Terrestrial Physics (ISTP) campaign. Two of these cameras share primary and some secondary optics and are designed to provide images of the nighttime auroral oval at visible wavelengths. A third camera is used to monitor the directions of the fields-of-view of these sensitive auroral cameras with respect to sunlit Earth. The auroral emissions of interest include those from $\mathrm{N}_{2}^{+}$ at $391.4 \mathrm{~nm}, \mathrm{O}$ I at 557.7 and $630.0 \mathrm{~nm}, \mathrm{HI}$ at $656.3 \mathrm{~nm}$, and $\mathrm{O} \mathrm{Il}$ at $732.0 \mathrm{~nm}$. The two auroral cameras have different spatial resolutions. These resolutions are about 10 and $20 \mathrm{~km}$ from a spacecraft altitude of $8 R_{e}$. The time to acquire and telemeter a $256 \times 256$-pixel image is about $12 \mathrm{~s}$. The primary scientific objectives of this imaging instrumentation, together with the in-situ observations from the ensemble of ISTP spacecraft, are (1) quantitative assessment of the dissipation of magnetospheric energy into the auroral ionosphere, (2) an instantaneous reference system for the in-situ measurements, (3) development of a substantial model for energy flow within the magnetosphere, (4) investigation of the topology of the magnetosphere, and (5) delineation of the responses of the magnetosphere to substorms and variable solar wind conditions.
\end{abstract}

\section{Introduction}

The first global images of Earth's aurora were acquired by three scanning photometers, one each for emissions from $\mathrm{N}_{2}^{+} 391.4 \mathrm{~nm}$, O I $557.7 \mathrm{~nm}$, and O I $630.0 \mathrm{~nm}$, on board the low-altitude spacecraft ISIS 2 (Anger et al., 1973; Shepherd et al., 1973). Although these images were obtained over the Northern Hemisphere only once per orbit because orbital motion provided one of the dimensions for the pixel array of an image, the potential value of global images for organizing and interpreting in-situ observations of particles and fields was clearly demonstrated. Indeed the ability to obtain global images provided considerable excitement in the scientific community from the visualization of the overall morphology of the auroral emissions (Anger et al., 1974). The ISIS-2 observations were soon followed by the availability of broad-band images from similarly scanning radiometers, $400-1130 \mathrm{~nm}$, from the Defense Meteorological Satellite Program (DMSP) spacecraft (Rogers et al., 1974). Because the ISIS-2 and DMSP satellites orbited at low-altitudes, in the range of $1000 \mathrm{~km}$, typically only a portion of the auroral oval was viewed during a single polar pass. These spacecraft were later followed by the launch of Kyokko into an orbit with apogee of about $4000 \mathrm{~km}$. Kyokko was equipped with an imagememory tube and obtained global auroral images at far-ultraviolet wavelengths,

Space Science Reviews 71: 297-328, 1995.

(C) 1995 Kluwer Academic Publishers. Printed in Belgium. 
in this case within a broad passband at about 120 to $140 \mathrm{~nm}$, with unprecedented temporal resolution (Kaneda et al., 1977). The frame repetition period was 2 min. In the 1980 s auroral imagers were launched with several spacecraft. These spacecraft were Dynamics Explorer 1 (Frank et al., 1981), HILAT (Meng and Huffman, 1984), Viking (Anger et al., 1987), Polar BEAR (Meng et al., 1987), and Akebono (Kaneda and Yamamoto, 1991). HILAT and Polar BEAR were launched into low-altitude orbits and thus yielded an auroral image once per orbital period, about $100 \mathrm{~min}$. However, their imaging equipment included spectrometers with sufficient resolution to separate the O I 130.4-nm and 135.6-nm emissions. Such spectroscopy is important in quantitative evaluation of the spectrum of precipitating electrons (Strickland et al., 1983). The scanning spectrometer on Polar BEAR also provided images of the nighttime auroral oval at visible wavelengths. The apogee of the Viking orbit was located at higher altitudes, about $13500 \mathrm{~km}$. The two cameras, one for the $\mathrm{OI}$ emissions at 130.4 and $135.6 \mathrm{~nm}$ with passband extending into the longer wavelengths of $\mathrm{N}_{2}$ Lyman-Birge-Hopfield (LBH) emissions and the other for the LBH emissions, were capable of frame repetition periods of $20 \mathrm{~s}$. These Viking images were very useful in studies of the temporal evolution of the spatial distribution of features at auroral and polar cap latitudes (Murphree et al., 1987).

The three spin-scan photometers on Dynamics Explorer 1 (DE 1) provided hundreds of thousands of global auroral images during the nine years following their initial operational turn-on in fall of 1981 (Frank and Craven, 1988). Two of these imagers viewed the aurora at visible wavelengths in narrow passbands as selected with a filter wheel. The third imager was equipped with broad-band filters for farultraviolet wavelengths in the range of $\sim 120$ to $175 \mathrm{~nm}$. The orbit was sufficiently high, an altitude of about $22000 \mathrm{~km}$, that the viewing time of the entire auroral oval during a single orbit was 2 to $3 \mathrm{hr}$. Such viewing times allowed continuous global viewing of the development of entire auroral substorms, i.e., through growth, onset, expansion and recovery phases. The imagers for visible wavelengths are the first, and at present only, optical systems that have successfully viewed the dim auroral emissions in the nighttime atmosphere with the intense emissions from sunlit Earth in the field-of-view. These imagers were usually operated in a mode such that an image frame with a field-of-view $30^{\circ} \times 30^{\circ}$ divided into 14400 pixels was acquired once each $720 \mathrm{~s}$.

The Visible Imaging System(VIS) for the Polar spacecraft is designed to achieve high-time and -spatial resolution images of the nighttime polar and auroral emissions at visible wavelengths. There is an ancillary camera for far-ultraviolet wavelengths within a broad passband, $124-149 \mathrm{~nm}$. This camera can provide full images of Earth from radial distances $>5.8 R_{e}$ and is used to verify the proper pointing of a two-axis targeting mirror for the two primary cameras for visible wavelengths. The optics for the visible cameras is based upon the off-axis catoptric design with super-polished surfaces that was successfully used for the DE-1 spin-scan imagers. Because the VIS is mounted on a despun platform and can stare at Earth its per- 
formance in terms of angular resolution and frame rate can be greatly improved relative to that for the serial single-pixel sampling on the rotating DE 1. For example, consider the viewing of the nighttime auroral zone from a Polar spacecraft altitude of $7.4 R_{e}$ and high latitude. The targeting mirror for the cameras for visible wavelengths can be used to position the fields-of-view of these cameras such that viewing of the auroras is optimized. For the low-resolution camera its field-of-view is $5.6^{\circ} \times 6.3^{\circ}$ and sufficient to usually include the entire nighttime auroral oval. Within this field- of-view a frame of 65500 pixels can be telemetered every $12 \mathrm{~s}$. For a DE-1 image taken at this altitude the corresponding frame of 576 pixels could be telemetered every $144 \mathrm{~s}$. The counts/pixel for a given auroral brightness are similar for the two images.

The scientific objectives for observations with VIS can be grouped into five primary categories: (1) quantitative assessment of the dissipation of magnetospheric energy into the auroral and polar ionospheres, (2) an instantaneous reference system for the in-situ measurements with the ISTP spacecraft, (3) development of a substantial model for energy flow within the magnetosphere, (4) investigation of the topology of the magnetosphere, and (5) delineation of the responses of the magnetosphere to substorms and variable solar wind conditions. It should be realized that these general objectives cannot be achieved without the in-situ observations from the various ISTP spacecraft. Much has been learned from previous studies as to the specific investigations that will contribute to these general objectives. Because this paper is devoted to a description of the instrumentation we limit our discussion of the objectives to an illustrative example for each category.

In order to achieve (1) above interleaved sequences of images of the emissions from $\mathrm{N}_{2}^{+}$at $391.4 \mathrm{~nm}$ and for $\mathrm{O}$ I at $630.0 \mathrm{~nm}$ are acquired. The 391.4-nm emission is a good measure of the electron energy flux into the atmosphere and the ratio of the two intensities is a measure of the electron energy spectral index. The complication for determination of the energy fluxes and electron spectra in this manner is caused by the reflectance of Earth's surface and, if present, clouds. This is basically a tractable radiative transfer problem (Rees et al., 1988). The reflectance of Earth and clouds and their contributions to the observed intensities are to be evaluated in part with images at filter wavelengths that are offset from auroral emission lines. The determination of electron energy spectra with the visible emission lines is complementary to that achieved at far-ultraviolet wavelengths and can be applied for lower electron energies than the latter measurement (Rees et al., 1988; Strickland et al., 1983). Of course, the visible observations can be only taken for the nighttime aurora where most of the precipitating charged particle energy fluxes occur whereas the far-ultraviolet measurements are possible for the sunlit atmosphere.

The provision of an instantaneous coordinate system, category (2), is obvious. The global auroral images place the in-situ observations in the context of auroral substorm phase or other activity and of geographical location of the imprint of charged particle precipitation. For example, measurements of particles and fields 
in the distant polar magnetosphere during periods that a theta aurora (Frank et al., 1986) is observed can resolve the controversy as to whether the transpolar arc of this auroral configuration is the footprint of bifurcation of the magnetospheric lobes (Frank, 1988) or large-scale spatial distortion of the plasma regimes in the magnetotail (Akasofu and Roederer, 1984; Lyons, 1985).

Category (3) studies of the gross flow of energy within the magnetosphere extend over a broad range, including the inference of the total magnetic energy in the magnetotail from the area poleward of the auroral oval and the relative motions of the ion and electron plasmas in the vicinity of the inner edge of the electron plasma sheet. In order to obtain the footprint of protons precipitating into the ionosphere the VIS is equipped with a narrow-band filter for $\mathrm{HI}$ 656.3$\mathrm{nm}$ emissions. With interlaced images of O I 557.7-nm emissions the large-scale interrelationship between precipitation of electrons and protons from the near-Earth plasma sheet into the atmosphere can be studied.

The mapping of plasma boundaries into features of the auroral luminosities is important for extending in-situ observations of these boundaries with a single spacecraft into a visualization of their geometries and temporal evolutions. Such studies are included in category (4). It is clear that such identification of these boundaries can contribute significantly to our knowledge of the magnetic field topology of the magnetosphere. Only limited studies of this type have been reported. One of the notable examples is the identification of poleward discrete arcs in the auroral oval with the plasma sheet boundary layer from simultaneous observations with the DE-1 imager and an ISEE-2 plasma analyzer (Frank and Craven, 1988) and with magnetometers on both spacecraft (Elphic et al., 1988). The comparison of images from the Polar spacecraft and in-situ fields and particles observations with both the Polar and Geotail spacecraft should substantially increase our knowledge of the magnetic topology of the magnetosphere and its relationship to major plasma regions.

Analysis of DE-1 image sequences for small, isolated substorms has revealed that the polar cap area, i.e., that area enclosed by the poleward edge of the auroral oval, responds to the southward turning of the interplanetary magnetic field (Frank and Craven, 1988; Frank, 1988). Although previous studies with lowaltitude observations indicated that this response occurs (Meng and Makita, 1986) the DE-1 images provided determination of the entire polar cap boundary with sufficient temporal resolution, $12 \mathrm{~min}$, to clearly identify this effect. These studies are part of the general topic (5) above. The DE-1 results showed that the polar cap area expands when the interplanetary field turns southward and increases until a substorm onset occurs. During the expansion phase the polar cap area decreases. The expansion and subsequent contraction of polar cap area can be interpreted as the storage and release, respectively, of the total magnetic energy in the magnetotail lobes. This energy can be quantitatively estimated with simple models of the magnetotail magnetic fields (Coroniti and Kennel, 1972). The question remains as to the precise connection between the polar cap area and open magnetic field lines 
in the lobes. Detailed analysis of auroral images and simultaneous fields and particles measurements with the Polar spacecraft should resolve this issue and refine the estimates of the transport of solar wind energy into the magnetotail and its explosive release during substorms.

The opportunity to construct and launch a state-of-the-art camera does not occur very often. With the addition of a few filters into the instrumentation the objectives can address several targets of opportunity. The two filters at 317.3 and $360.1 \mathrm{~nm}$ provide high spatial resolution for the total columnar ozone in Earth's sunlit atmosphere. One of these filters can be used to acquire global monitoring of the occurrence of lightning. A narrowband filter at $589.0 \mathrm{~nm}$ is also included for surveys of the Moon's Na cloud (Mendillo et al., 1991) and $\mathrm{Na}$ emissions in Earth's nighttime atmosphere. The filter for $\mathrm{OH}$ emissions at $308.5 \mathrm{~nm}$ can be used to pursue the topic of atmospheric holes by a search for clouds of $\mathrm{OH}$ above and in the upper atmosphere (Frank and Sigwarth, 1993).

\section{Overview of Instrumentation}

A photograph of the instrumentation is shown in Plate 1. The instrument is to be mounted on the despun platform of the Polar spacecraft. The top covers are removed. These covers are radiators equipped with optical surface reflectors (OSRs) for passively cooling the charge-coupled devices (CCDs) and the electronics. A diagram of the instrument as viewed from this top side is shown in Figure 1 and is useful in identifying the various subsystems that are visible in Plate 1. Two major compartments are visible in Plate 1, the optics section on the left-hand side and the electronics section on the right. The gold-colored aperture door and two rectangular collimators extend forward of the main optics section. The motordriven door provides protection for the internal optics against particulate material and condensables during launch and inflight reorientations of the spacecraft spin axis. The instrument is assembled in a Class- 100 clean room. The rectangular collimator on the left-hand side services the two cameras for auroras at visible wavelengths. The field-of-view provided by this collimator is $20^{\circ} \times 20^{\circ}$. These two cameras share primary optics and some of the secondary optics. The cameras are nearly identical with the major exception of angular resolution. The angular resolution of the medium-resolution camera is $0.011^{\circ} \times 0.013^{\circ}$ (pixel size) and that of the low-resolution camera is $0.022^{\circ} \times 0.025^{\circ}$. The instantaneous fields-of-view of these cameras is significantly less than that provided by the collimator. Thus a bi-axially rotated mirror is employed to cover this entire field-of-view by mosaicing images, particularly at low altitudes. An overview of the performance parameters for the cameras is given in Table I. At the primary focal plane a field-stop wheel is used to block the image of sunlit Earth from the secondary optics. This field stop wheel thusly prevents this intense light from direct entry into the secondary optics. The optical path is then folded in a complex geometry to accommodate the 
TABLE I

Overview of the visible imaging system (VIS)

\begin{tabular}{|c|c|c|}
\hline Size & \multicolumn{2}{|l|}{$61 \times 66 \times 25 \mathrm{~cm}$} \\
\hline Mass & \multicolumn{2}{|l|}{$28.65 \mathrm{~kg}$} \\
\hline Power & \multicolumn{2}{|l|}{$30 \mathrm{~W}$} \\
\hline Image frame rate & \multicolumn{2}{|l|}{$12 \mathrm{~s}$} \\
\hline Telemetry & \multicolumn{2}{|l|}{11 kilobits s $^{-1}$} \\
\hline Number of pixels/frame & \multicolumn{2}{|l|}{$256 \times 256$ pixels } \\
\hline Aurora cameras & Low resolution & Medium resolution \\
\hline \multicolumn{3}{|l|}{ Field-of-view } \\
\hline - Overall & $20^{\circ} \times 20^{\circ}$ & $20^{\circ} \times 20^{\circ}$ \\
\hline - Instantaneous & $5.4^{\circ} \times 6.3^{\circ}$ & $2.8^{\circ} \times 3.3^{\circ}$ \\
\hline Passband width ( 12 filters) & $\sim 1 \mathrm{~nm}$ & $\sim 1 \mathrm{~nm}$ \\
\hline Wavelength range & $308-732 \mathrm{~nm}$ & $308-732 \mathrm{~nm}$ \\
\hline Sensitivity $(630 \mathrm{~nm})$ & 3 counts kR ${ }^{-1}$ pixel $^{-1}$ & 0.8 count $\mathrm{kR}^{-1}$ pixel $^{-1}$ \\
\hline Time resolution & $12 \mathrm{~s}$ & $12 \mathrm{~s}$ \\
\hline Angular resolution & $0.02^{\circ}$ & $0.01^{\circ}$ \\
\hline \multicolumn{3}{|c|}{ Spatial resolution (at Earth's surface as seen from the apogee altitude of $8 R_{e}$ ) } \\
\hline & $20 \mathrm{~km}$ & $10 \mathrm{~km}$ \\
\hline \multicolumn{3}{|l|}{ Earth camera } \\
\hline Field-of-view & \multicolumn{2}{|l|}{$20^{\circ} \times 20^{\circ}$} \\
\hline Passband width & \multicolumn{2}{|l|}{$\sim 25 \mathrm{~nm}$} \\
\hline Wavelength range & \multicolumn{2}{|l|}{$124-149 \mathrm{~nm}$} \\
\hline Sensitivity & \multicolumn{2}{|l|}{7.2 counts $\mathrm{kR}^{-1} \mathrm{pixel}^{-1}$} \\
\hline Time resolution & \multicolumn{2}{|l|}{$12 \mathrm{~s}$} \\
\hline Angular resolution & \multicolumn{2}{|l|}{$0.08^{\circ}$} \\
\hline \multicolumn{3}{|c|}{ Spatial resolution (at Earth's surface as seen from the apogee altitude of $8 R_{e}$ ) } \\
\hline & \multicolumn{2}{|c|}{$70 \mathrm{~km}$} \\
\hline
\end{tabular}

allowed dimensions of the housing. A plane mirror rotated by a motor is used to determine which of the two cameras receives the image. The light is collimated and passed through a selected narrow-band filter (the gold-colored wheel) and the image is reformed at the faceplate of an image intensifier. The intensified image is then optically transferred to a CCD. This mechanical isolation allows the cooling of the CCD to temperatures in the range of $-90^{\circ} \mathrm{C}$ in order to obviate the deleterious effects of damage from energetic ions in the inner radiation zone. At these temperatures, the electrons associated with the displacement defects are trapped. The small gold-colored 'knobs' provide the heat strap connection to the topside radiator. 


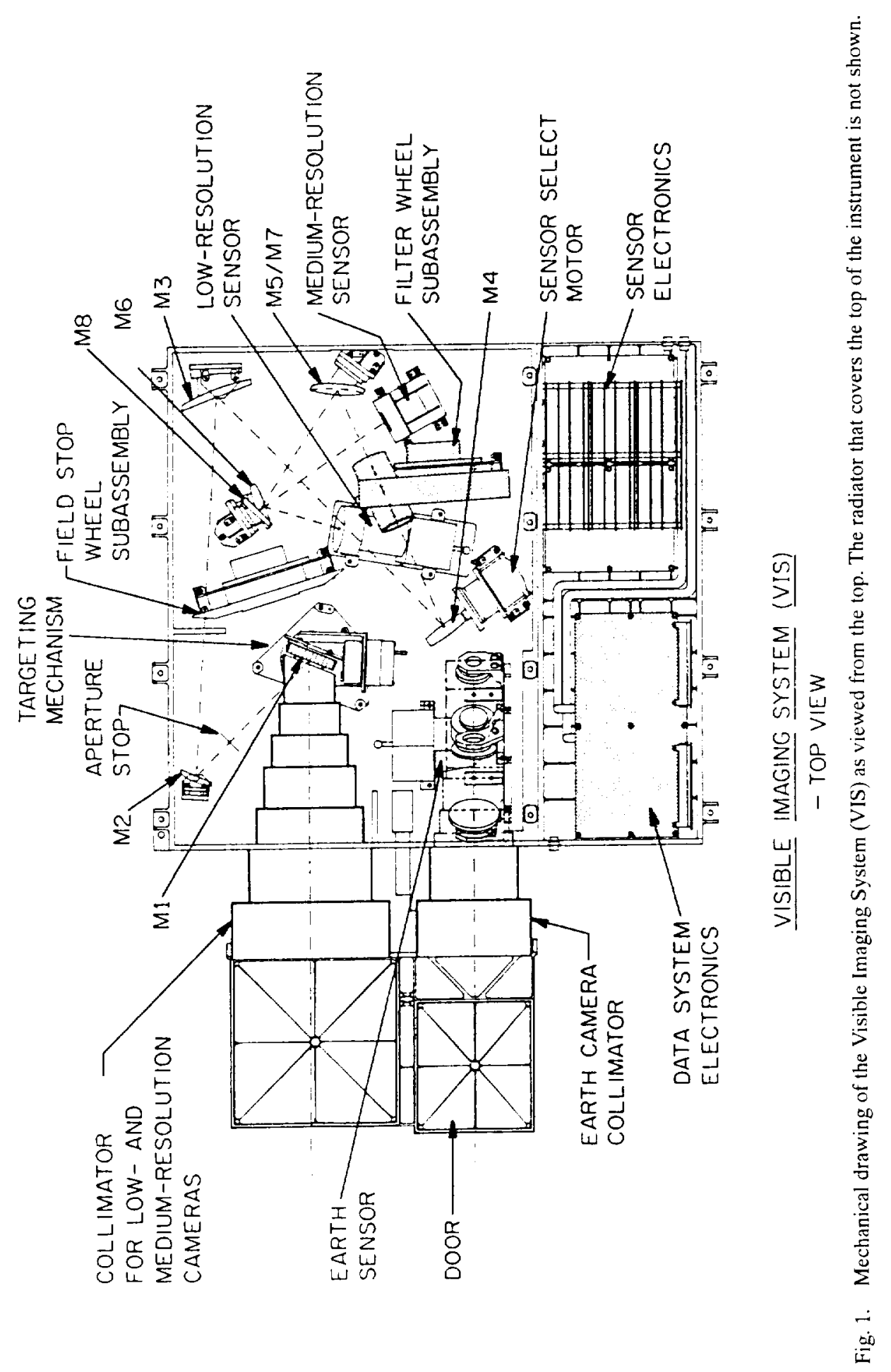




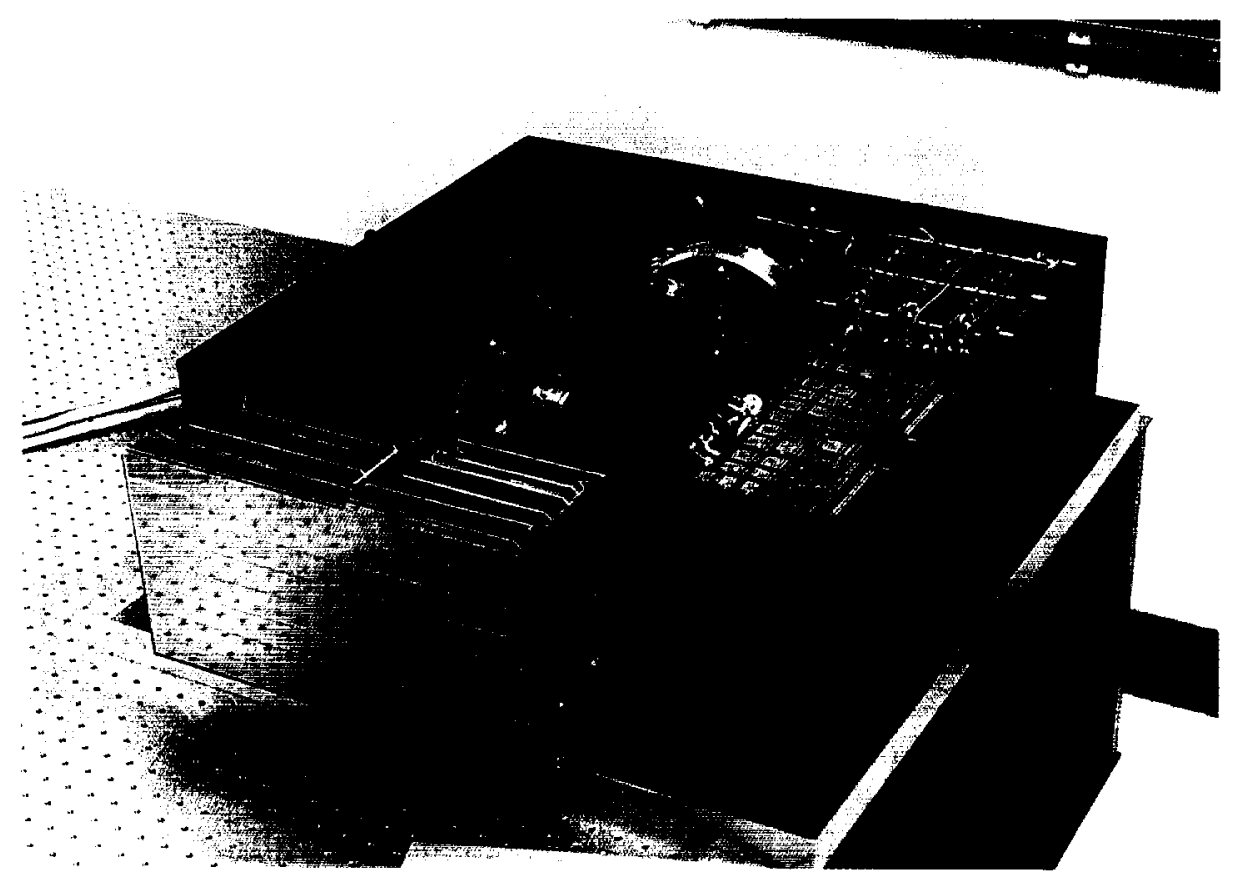

Plate 1. Photograph of the Visible Imaging System (VIS) without the top radiator plates.

The smaller rectangular collimator behind the right-hand side of the door is used for the Earth camera. This Earth camera provides a $20^{\circ} \times 20^{\circ}$ image without the need for mosaicing several images. The angular resolution is lesser than that of the visible cameras, $0.08^{\circ} \times 0.08^{\circ}$. For comparison the corresponding pixel for the imagers on DE-1 is circular with angular diameter $0.25^{\circ}$. The Earth camera is equipped with one broad-band filter at far-ultraviolet wavelengths. These images are processed within the VIS to ascertain that no intense light sources, such as sunlit Earth, are viewed by the secondary optics of the visible cameras. It is possible to telemeter one image from the Earth camera each $12 \mathrm{~s}$.

The electronics compartment is on the right-hand side of the instrument as viewed in Plate 1 . The electronics stack in the rear of this compartment is three identical sets of power supplies and control electronics for the three sensors. The front stack includes the six microprocessors with a total of 736 kbytes of memory for operating the instrument and for data compression. The two primary power convertors are located out-of-sight below the sensor electronics. 


\section{Mechanical Design}

The VIS mechanical structure consists of three major subassemblies; the optics housing, the electronics housing, and the radiator. In addition the various motors that are employed in the instrument are described in this section.

The optics housing is designed to provide a stiff, stable optics platform for the two visible cameras and the Earth camera. This housing is required to be light-tight and to be of minimum mass. The optics housing assembly includes the optical bench, optical subassemblies, and the collimator extension subassemblies. The optical subassemblies are mounted to a pocketed beryllium base plate that serves as the optical bench. The structure is optimized for the reduction of thermal gradients.

The optical bench is enclosed by light-tight, pocketed $\mathrm{Mg}$ walls. Each interface, i.e., optics wall-to-bench and optics wall-to-wall, is baffled and sealed with $\mathrm{O}$ rings. To prevent the possibility of distortion of the optical bench by the difference in the thermal expansion coefficients of $\mathrm{Be}$ and $\mathrm{Mg}$, attach points are designed to eliminate fastener interference.

The collimator extensions include the Earth-camera collimator, the collimator for the two visible cameras, and an aperture door for the collimators. Each collimator is machined from $\mathrm{Mg}$ stock and subsequently etched to minimize mass. The baffles in the collimators are coated with Martin Black. Each of the two collimators is thermally isolated from the optics housing. A special light-tight seal is used to prevent entry of stray light into the optics housing, without metal-to-metal contact at this interface. The aperture door is machined from a thick $\mathrm{Mg}$ plate to produce a stiff, low-mass structure.

The electronics housing is fabricated from $\mathrm{Mg}$ and is attached to the optics housing by a light-tight interface wall which also accommodates the electrical interface between the optics and electronics sections. Because this housing interfaces with the optics housing, the radiator, and the spacecraft platform, careful consideration is given to the structural dynamics of these interfaces in order to prevent distortion of the optical bench.

The instrument thermal radiator consists of two thermally isolated plates that are covered with OSRs. One of these radiators is used to dispose of the thermal energy from the power dissipation in the optics and electronics sections and to maintain the temperature of the optical bench in the range -20 to $0{ }^{\circ} \mathrm{C}$. The larger radiator is used to passively cool the CCDs to $-90^{\circ} \mathrm{C}$. This radiator is coupled directly to the tantalum radiation shield surrounding each $\mathrm{CCD}$ by means of a copper heat strap.

There are six stepping motors and one wax motor in the VIS. The six motors are manufactured by Schaeffer Magnetics, Inc. and are two similar types. The first type is used for the bi-axial targeting mirror (two motors), the sensor select mirror, and the collimator door. The second design is used for the filter wheel and the field stop wheel. Both motors are similar except that the motor step for the first 
type is $3.75^{\circ}$ and is reduced by an integral $80: 1$ harmonic drive, and the motor step for the second type is $1.5^{\circ}$ with no harmonic drive. Both motor types operate efficiently at 100 steps $\mathrm{s}^{-1}$ and have the capability of bidirectional rotation. The two motors for the bi-axial targeting mirror provide motion of the fields-of-view for the visible cameras with respect to the platform in two orthogonal planes, i.e., in a plane parallel to the spacecraft spin axis and in a plane perpendicular to this axis. The increments in the field-of-view are $0.094^{\circ}$ in either direction. The Starsys wax motor is used for a one-shot mechanism that permanently opens the collimator door in the event that the door motor is not operable.

\section{Optics for the Low- and Medium-Resolution Cameras}

The Visible Imaging System (VIS) was designed with a stringent set of specifications in order to image the dim nightside auroras with the bright dayside of Earth within the instrument's field-of-view. The VIS is an $f / 8$ re-imaging system with an intermediate primary focal plane and final focal planes at the sensors. The camera employs off-axis parabolic and flat mirrors that provide the image at either the low-resolution or medium- resolution sensors.

Exploded views of the VIS in the low-resolution and medium-resolution configurations are shown in Figures 2 and 3, respectively. Light enters the collimator assembly and is reflected by flat mirror M1 which is mounted on a bi-axial targeting mechanism. The targeting mechanism is driven by two motors. Operation of this bi-axial targeting mirror assembly permits the acquisition of $5.4^{\circ} \times 6.3^{\circ}$ or $2.8^{\circ} \times 3.3^{\circ}$ images at any position within the $20^{\circ} \times 20^{\circ}$ field-of-view of the collimator for the low-resolution and medium-resolution cameras, respectively. An aperture stop is placed between M1 and the off-axis parabolic reflector M2. The aperture stop is also the entrance pupil with a diameter of $2.0 \mathrm{~cm} . \mathrm{M} 2$ provides the primary image at the position of the field stop wheel. The selection of field stops on the wheel allows imaging near Earth's terminator, i.e., rejection of those portions of the primary image with sunlit Earth from entering the secondary optics. In the secondary optics the light is reflected by an angle of $\sim 41^{\circ}$ by the off-axis parabolic surface of M3 and becomes nearly collimated. Sensor select mirror M4 is used to direct the light into the optical path for either the low-resolution or medium-resolution sensors.

For the low-resolution sensor the nearly collimated light passes through a Lyot stop and subsequently through one of twelve filters in the filter wheel. Off-axis parabolic reflector M5 and the plane turning-mirror M6 present the focused image at the low-resolution sensor (see Figure 2). For the medium-resolution sensor the nearly collimated light from M4 passes through another Lyot stop and then through the filter wheel (see Figure 3). Off- axis parabolic reflector $M 7$ and inverted parabolic reflector M8 provide the image at the medium-resolution sensor. The inverted parabolic reflector M8 magnifies the image by a factor of two relative to 
the image at the low-resolution sensor. The optical properties of each of the eight mirrors are summarized in Table II.

The resolution of the optics for the low-resolution and medium-resolution cameras is summarized by the 'spot' diagrams in Figure 4. These spot diagrams are given at the position of the respective sensor input planes and for the four corners and center of the respective fields-of-view. For comparison, the linear dimension of one pixel at the sensor input image plane for the $256 \times 256$-pixel format is shown. The modulation transfer functions (MTFs) of the optics, exclusive of the sensor, are given in Table III for the low- and medium-resolution cameras. The diffraction limit is also specified.

The mirrors were manufactured by Speedring Systems, Inc. of Rochester Hills, Michigan. With the exception of M1 which is Zerodur, each mirror is a beryllium substrate that is diamond turned, coated with electroless nickel, and subsequently lapped to final figure with a low-scatter super-polish with surface roughness of $\leq 1 \mathrm{~nm}$. Vapor-deposited aluminum is used to increase the reflectivity to greater than $90 \%$, and an overcoat of $\mathrm{SiO}_{2}$ is applied to protect the surface from degradation. The mirrors are mounted with beryllium brackets to a beryllium optical base plate to achieve an athermal design. Thermal analysis shows that temperature differences should be less than $10^{\circ} \mathrm{C}$ and will not significantly affect the alignment of the optical elements.

\section{Stray Light Rejection}

An important consideration in the design of the VIS is the adequate rejection of stray light. At visible wavelengths the sunlit Earth can be brighter by a factor of $10^{6}$ than the dim nightside auroral emissions. One of the most important features of the VIS for the rejection of stray light is the low-scatter super-polished mirrors. The values for their bidirectional reflectance distribution function (BRDF) are $10^{-2}$, $3 \times 10^{-4}, 6 \times 10^{-5}$, and $8 \times 10^{-6} \mathrm{sr}^{-1}$ for angles of $1^{\circ}, 3.5^{\circ}, 10^{\circ}$, and $45^{\circ}$ from the specular direction, respectively. The corresponding BRDF values for the Zerodur M1 mirror are $5 \times 10^{-4}, 2 \times 10^{-5}, 1 \times 10^{-5}$, and $3 \times 10^{-6} \mathrm{sr}^{-1}$, respectively. A further important aspect of the design is the use of off-axis optical elements to ensure that no mechanical structure intrudes into the primary or secondary optical paths. Such intrusion can cause sufficient scattering to overwhelm the intensities of nightside auroral emissions.

There are three critical stops in the optics. The aperture stop rejects all rays from outside the fields-of-view at the sensors, e.g., from the collimator baffle edges and M1 mirror mount. Secondly a Lyot stop is located between mirror M4 and the filter at the position corresponding to the image of the aperture stop. This Lyot stop rejects stray light scattered from the internal baffles between the aperture stop and itself and from the edges of the aperture stop. The third critical stop is the field stop at the primary image plane. For images taken when viewing near the terminator 


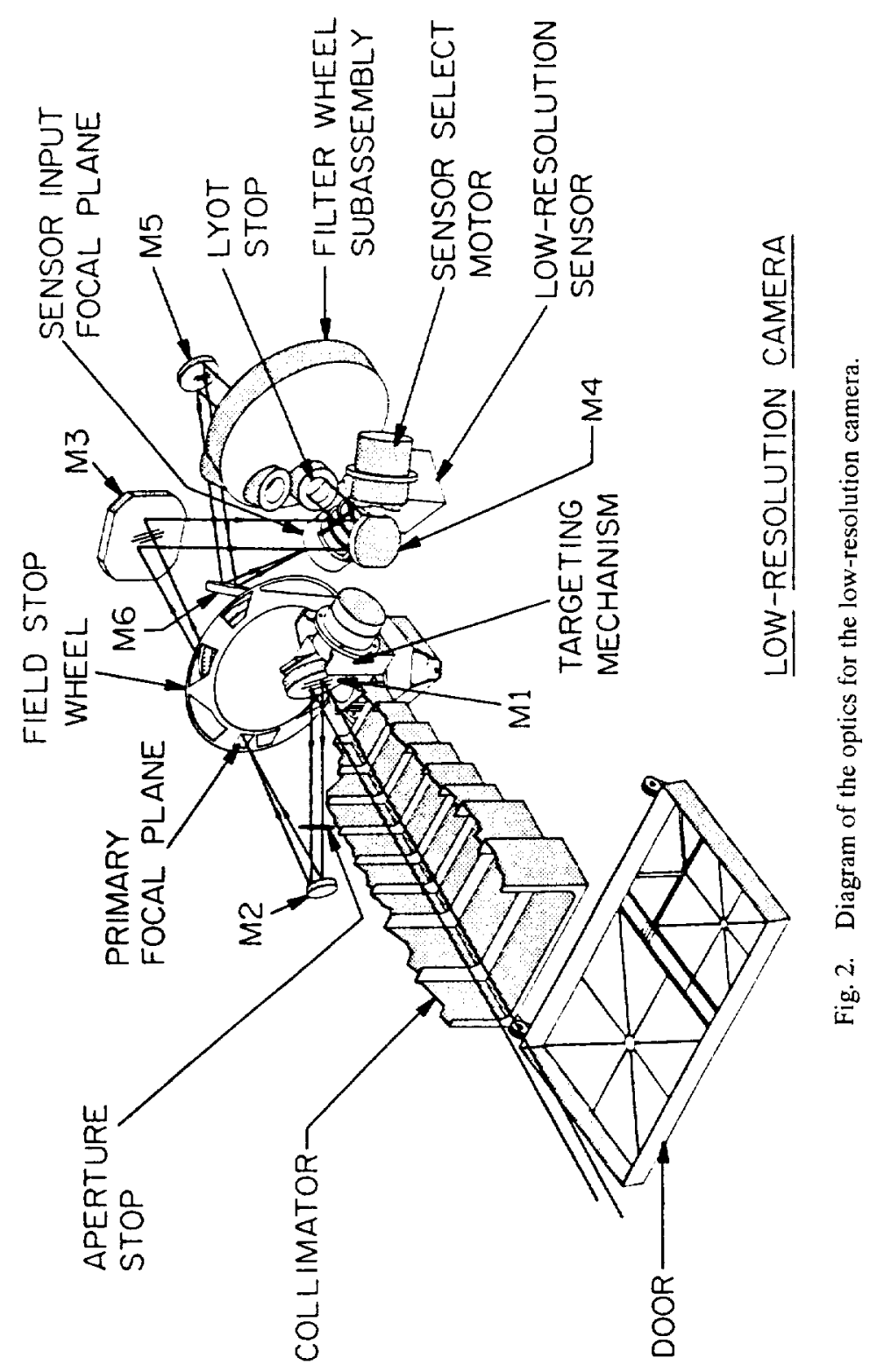


TABLE II

Optical elements of the low- and medium-resolution cameras

\begin{tabular}{lllll}
\hline $\begin{array}{l}\text { Mirror, function } \\
\text { (description) }\end{array}$ & \multicolumn{4}{l}{ Clear aperture } \\
\cline { 2 - 5 } & $\begin{array}{l}\text { Area } \\
\left(\mathrm{cm}^{2}\right)\end{array}$ & $\begin{array}{l}\text { Radius, } r \\
(\mathrm{~cm})\end{array}$ & $\begin{array}{l}\text { Focal } \\
\text { length } \\
(\mathrm{cm})\end{array}$ & $\begin{array}{l}\text { Off-axis } \\
\text { distance, } H \\
(\mathrm{~cm})\end{array}$ \\
\hline $\begin{array}{l}\text { M1, targeting } \\
\text { (Flat) }\end{array}$ & 22.7 & 2.69 & Infinity & - \\
$\begin{array}{l}\text { M2, primary focusing } \\
\text { (Off-axis parabola) }\end{array}$ & 7.5 & 1.55 & 17.000 & 12.441 \\
$\begin{array}{l}\text { M3, collimating } \\
\text { (Off-axis parabola rectangular) }\end{array}$ & 62.4 & $3.9 \times 4.0$ & 25.000 & 17.365 \\
$\begin{array}{l}\text { M4, sensor select } \\
\text { M5, low resolution } \\
\text { (Off-axis parabola) }\end{array}$ & 19.6 & 2.5 & Infinity & - \\
$\begin{array}{l}\text { M6, low resolution turning } \\
\text { (Flat oval) }\end{array}$ & 19.6 & 2.5 & 18.750 & 23.140 \\
$\begin{array}{l}\text { M7, medium resolution } \\
\text { (Off-axis parabola) }\end{array}$ & 15.7 & $2.0 \times 2.5$ & Infinity & - \\
$\begin{array}{l}\text { M8, medium resolution magnifying } \\
\text { (Off-axis inverted parabola) }\end{array}$ & 12.6 & 2.0 & -17.790 & 7.477 \\
\hline
\end{tabular}

an appropriately shaped stop is selected to prevent the image of sunlit Earth from entering the secondary optical path.

Narrow-band filters are also used to suppress scattered light. Their full-width half- maximum passbands are typically about 1 to $2 \mathrm{~nm}$. The out-of-band rejection is $10^{-6}$. The temperatures of the filters are actively controlled by heaters and are maintained at $5( \pm 1){ }^{\circ} \mathrm{C}$ to obviate the variations of peak transmission wavelength with temperature.

Coatings and knife-edged baffles are carefully used in the instrument to aid in the rejection of stray light. The baffle edges in the collimator and to the position of the field stop are coated with Martin Black to minimize the scattering. Martin Black absorbs $99.5 \%$ of the incident light which can be compared to the $85 \%$ absorptions for Dow 9/40 and black anodization. The interior sidewalls of the collimator and the internal walls of the instrument are coated with Dow 9/40. The aperture door is an integral part of the collimator and partially shields the front baffling from illumination by the sunlit Earth. 


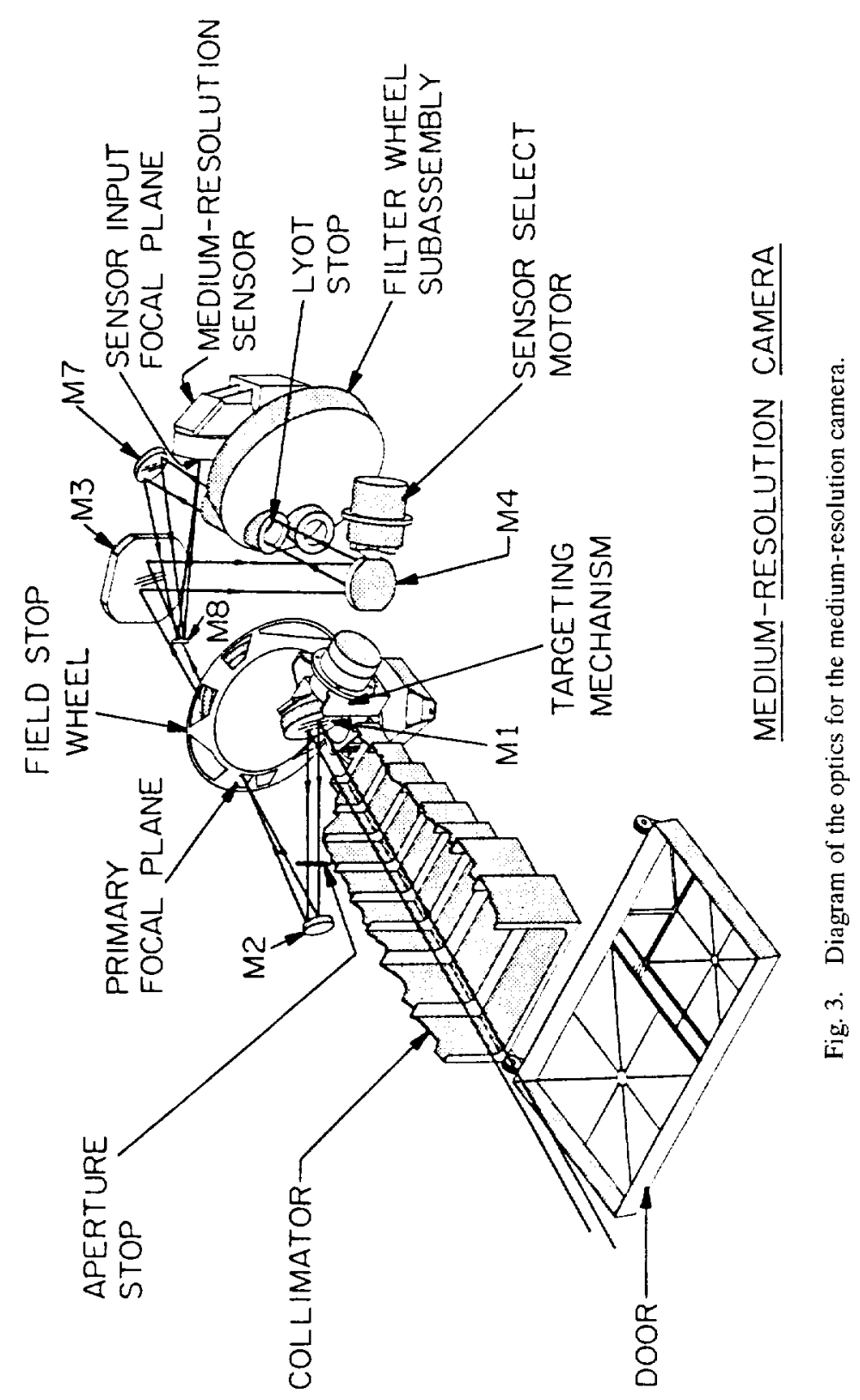




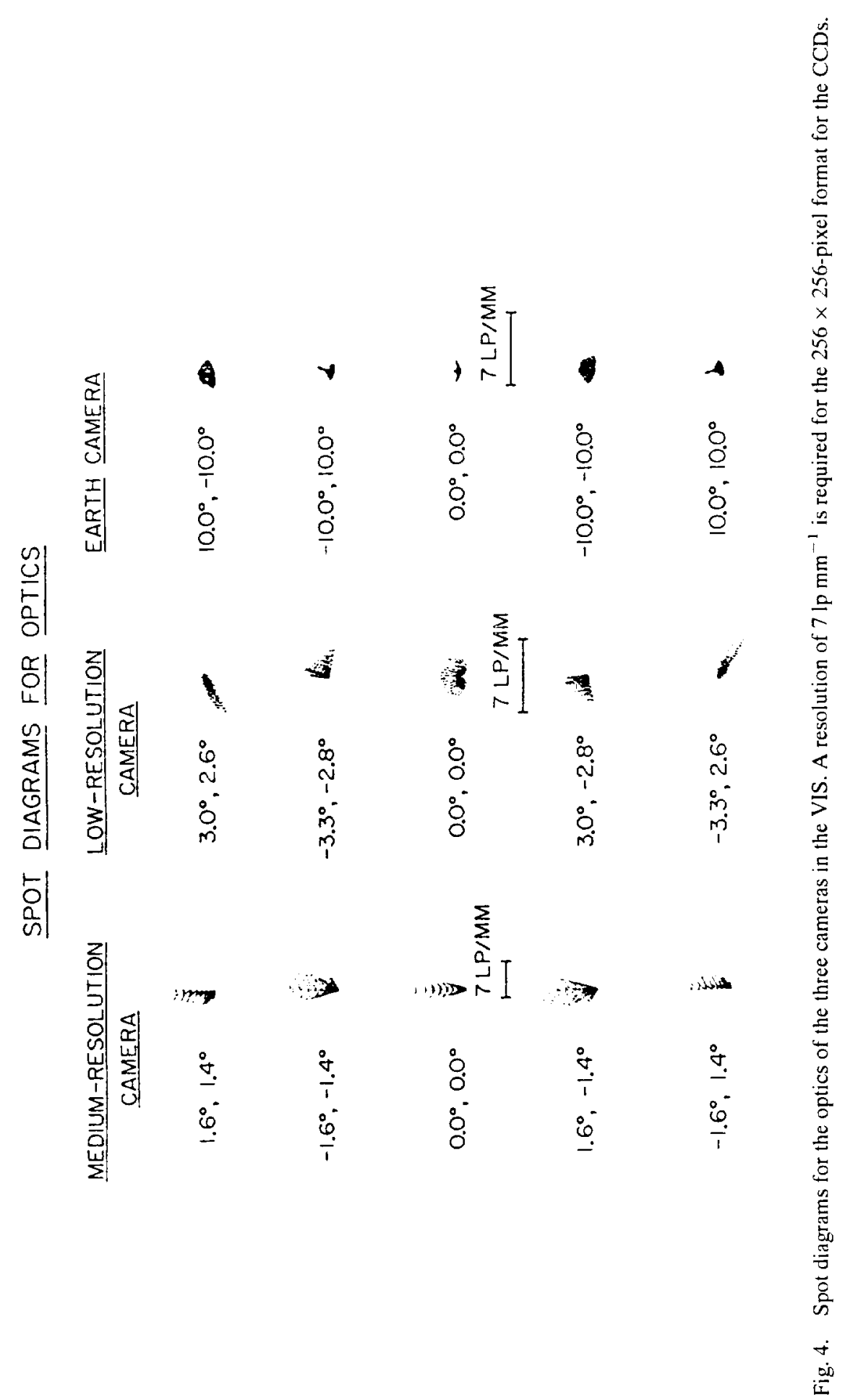


TABLE III

MTF for camera optics (diffraction limit)

\begin{tabular}{rlll}
\hline Resolution & $\begin{array}{l}\text { Low-resolution } \\
\text { camera }\end{array}$ & $\begin{array}{l}\text { Medium-resolution } \\
\text { camera }\end{array}$ & $\begin{array}{l}\text { Earth } \\
\text { camera }\end{array}$ \\
\hline $7 \mathrm{lp} \mathrm{mm} \mathrm{m}^{-1}$ & $0.88(0.95)$ & $0.57(0.90)$ & $0.96(0.99)$ \\
$14 \mathrm{lp} \mathrm{mm}$ & $0.72(0.90)$ & $0.28(0.80)$ & $0.87(0.98)$ \\
\hline
\end{tabular}

\section{Earth-Camera Optics}

The purpose of the Earth camera is real-time monitoring of the fields-of-view of the low- and medium-resolution sensors in order to determine that the sunlit Earth is not being viewed by these latter sensitive sensors. Thus the Earth camera is used to prevent exposure and subsequent gain degradation due to the extremely high light intensities. In practice this is accomplished by determining in real time the position of the planetary limb with the Earth camera and cross-checking this location with that expected from onboard tables based on predictions used in operations planning. If these predicted locations are in disagreement with those observed with the Earth camera then the visible imaging sensors are safed and the images from the Earth camera are telemetered in order to trace the source of the discrepancy.

The optics of the Earth camera consists of three off-axis mirrors. This design is based upon a similar camera previously described by Hallam et al. (1983). The present optics provides a speed of $f / 4.6$ with an entrance pupil diameter of $0.85 \mathrm{~cm}$. A diagram of the mechanical configuration of the Earth sensor is shown in Figure 5. A list of the mirror parameters is given in Table IV. The image at the sensor is provided by a convex spherical mirror MES1, an off-axis parabolic mirror MES2 and a concave spherical mirror MES3, in order of their appearance along the optical path. An ultraviolet transmission interference filter with peak transmission at $130 \mathrm{~nm}$ is placed in front of the sensor. The half-maximum full-width of the passband is $25 \mathrm{~nm}$.

The field-of-view of the Earth camera is $20^{\circ} \times 20^{\circ}$. For reference the full angle of Earth as seen from an altitude of $8 R_{e}$ is $12.8^{\circ}$. The angular resolution of the optics is shown in the Figure 4. These spot distributions are shown for the four corners and the center of the field-of-view. The linear dimension for one pixel, in the $256 \times 256$-pixel format, at the sensor input image plane is shown for comparison. The distortion of the image is to be removed with ground processing of the image. This primarily barrel distortion is minimal for the large field-of-view of the camera and is displayed in Figure 6. The MTF of the optics of the Earth camera is given in Table III. In the $256 \times 256$-pixel format the corresponding spatial frequency is 7 line pairs $\mathrm{mm}^{-1}$. The MTF at 7 line pairs $\mathrm{mm}^{-1}$ is 0.96 . 


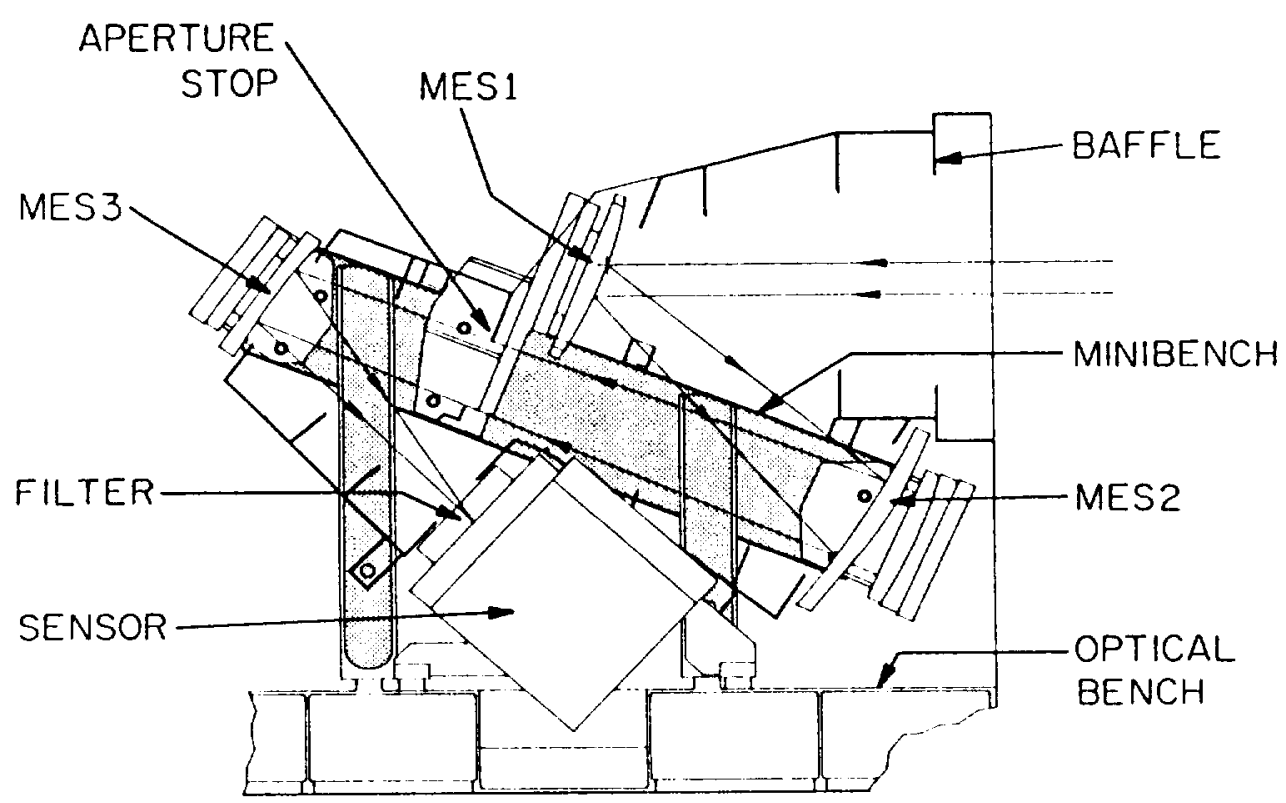

EARTH CAMERA

Fig. 5. Diagram of the optics for the Earth camera.

TABLE IV

Optical elements of the Earth camera

\begin{tabular}{lllll}
\hline $\begin{array}{l}\text { Mirror } \\
\text { (description) }\end{array}$ & $\begin{array}{l}\text { Area } \\
\left(\mathrm{cm}^{2}\right)\end{array}$ & $\begin{array}{l}\text { Radius, } r \\
(\mathrm{~cm})\end{array}$ & $\begin{array}{l}\text { Focal length } \\
(\mathrm{cm})\end{array}$ & $\begin{array}{l}\text { Off-axis distance } \\
H(\mathrm{~cm})\end{array}$ \\
\hline $\begin{array}{l}\text { MES1 } \\
\text { (Spherical convex) }\end{array}$ & 15.2 & 2.2 & -6.515 & - \\
$\begin{array}{l}\text { MES2 } \\
\text { (Off-axis parabola) }\end{array}$ & 26.4 & 2.9 & 12.917 & 3.996 \\
$\begin{array}{l}\text { MES3 } \\
\text { (Spherical concave) }\end{array}$ & 11.3 & 1.9 & 11.794 & - \\
\hline
\end{tabular}

The mirrors for the Earth camera are constructed from a beryllium substrate with a super-polished electroless nickel coating and a final overcoat of $\mathrm{MgF}_{2}$. The mirror figure is $\lambda / 8$ peak-to-peak at visible wavelengths. The optical elements are mounted on a beryllium optical minibench. The optical axis of the Earth camera is co-aligned with that of the collimator for the cameras for visible wavelengths. Stray 
EARTH CAMERA

- IMAGE DISTORTION

AT OPTICS FOCAL PLANE

- - OBJECT — IMAGE

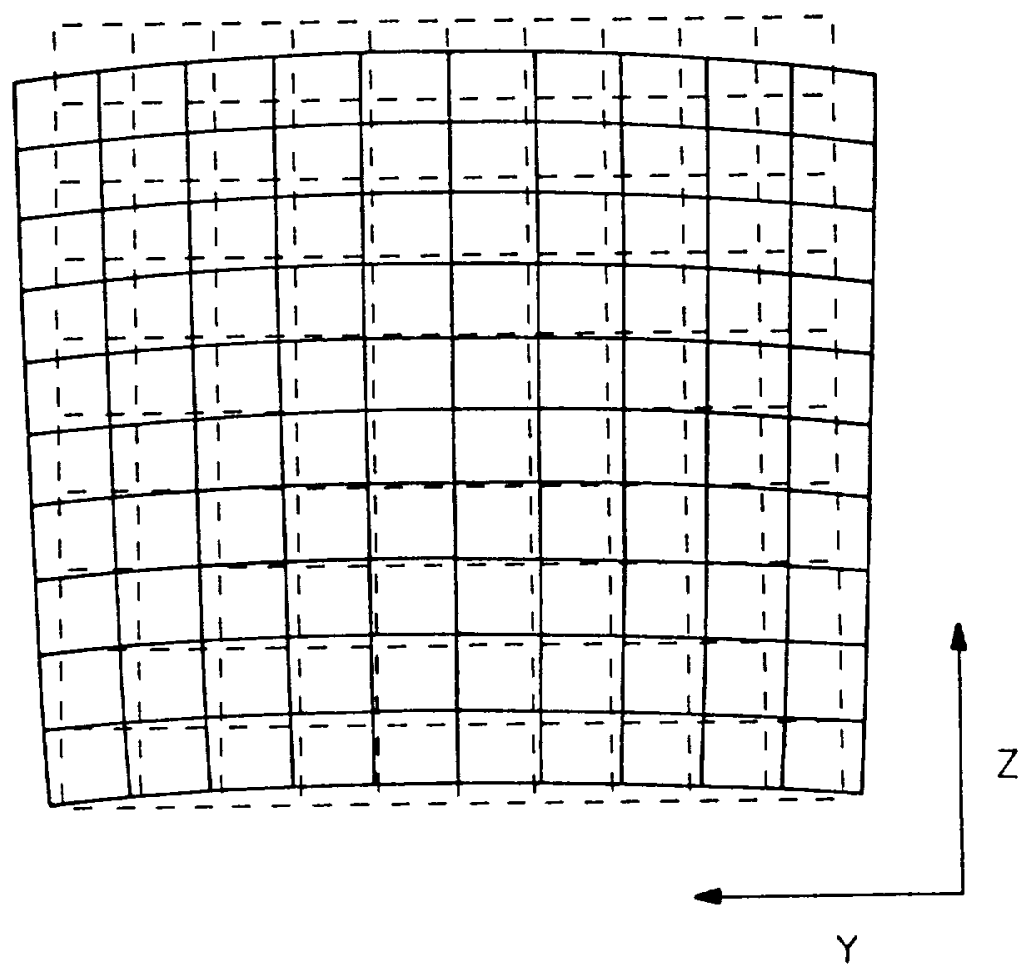

Fig. 6. Image distortion for the Earth-camera optics at the input focal plane of the sensor assembly.

light scattering is suppressed to negligible values by the off-axis optical elements, super-polished mirror surfaces, transmission filter with aluminum flashing, baffled collimator, and internal baffles. The stray light intensity is significantly lesser than that for the visible cameras because the far-ultraviolet intensities from sunlit Earth are generally greater by factors of only about 1000 or less than those from a dim nightside aurora.

\section{Sensors}

The VIS is equipped with three sensor assemblies, one each for the two cameras for visible wavelengths and the third for the Earth camera. A diagram of the sensor for the low-resolution visible camera is shown in Figure 7. The three sensors are 
similar with the exception of photocathode and a folding mirror in the other two sensor assemblies.

The image intensifiers are based in large part on the design for commercial generation-II 25-mm devices. In order to increase the photocathode lifetime a thin film of $\mathrm{SiO}_{2}$ is deposited over the front face of the microchannel plate (MCP) assembly. This assembly consists of two MCPs in the chevron configuration. The photocathode for the visible cameras is trialkali type S20 and that for the Earth camera is cesium iodide. The output electrons from the MCPs are proximityfocused into a phosphor, JEDEC registration $\mathrm{GH}$. The wavelength for the phosphor spectral maximum is $525 \mathrm{~nm}$. The decay times for the phosphor emissions after shuttering the light source to the sensor are about $250 \mu \mathrm{s}$ and $1 \mathrm{~s}$ for decreases by factors of $10^{-2}$ and $10^{-3}$, respectively. The image intensifiers were manufactured by Science Applications International Corporation at San Diego and the MCPs were supplied by Galileo Electro-Optics Corporation. The overall amplification of the light input is in the range of $10^{5}$ and is determined by the potential differences across the MCP assembly and between the MCP output face and the phosphor. These bias voltages are controlled by ground command and can be selected to values of 0 to $2500 \mathrm{~V}$ and 0 to $5700 \mathrm{~V}$, respectively. The gain of the image intensifier is sufficient to allow single-electron events at the entrance aperture of the MCP to be recorded by the CCD. The photocathodes can be back-biased in order to prevent current surges in the MCPs due to an extremely bright light source or to eliminate the effects of stray light from the science antennas that are mounted on the spinning section of the spacecraft and hence periodically appear within the fields-of-view of the cameras. This electronic shutter can be activated by an alarm from the MCP current monitor and by spin phase information from the spacecraft that is processed by the instrument electronics.

The image on the phosphor at the exit face of the intensifier is transferred to the CCD by a coupling lens assembly. A series of baffles prevents significant stray light scattering from the phosphor into the $\mathrm{CCD}$ (see Figure 7). For the sensor assemblies of the medium-resolution camera and Earth camera a folding mirror immediately follows the image intensifier in order to rotate the optical path by $90^{\circ}$ for mechanical accommodation. A wideband transmission filter is mounted on the back of the coupling lens subassembly in order to limit chromatic aberration to acceptable values. The center of the filter passband and the passband width at halfmaximum transmissions are $530( \pm 25) \mathrm{nm}$. The coupling lenses are followed by a radiation plug subassembly, a $2-\mathrm{cm}$ thick meniscus lens that can be mechanically translated along the chief ray for image focusing at the CCD.

The CCD is a Photometrics model PM516A which is manufactured by the LoralFairchild Imaging Sensors Group. This device has a pixel size of $20 \times 20 \mu \mathrm{m}$ with a $2-\mu \mathrm{m}$ channel stop. The array size is $512 \times 512$ pixels. The baseline operating mode sums a block of $2 \times 2$ pixels to yield one pixel. The design of the CCD electronic controller is centered upon a custom-made type- 8000 gate array fabricated by United Technologies Microelectronics Center. 


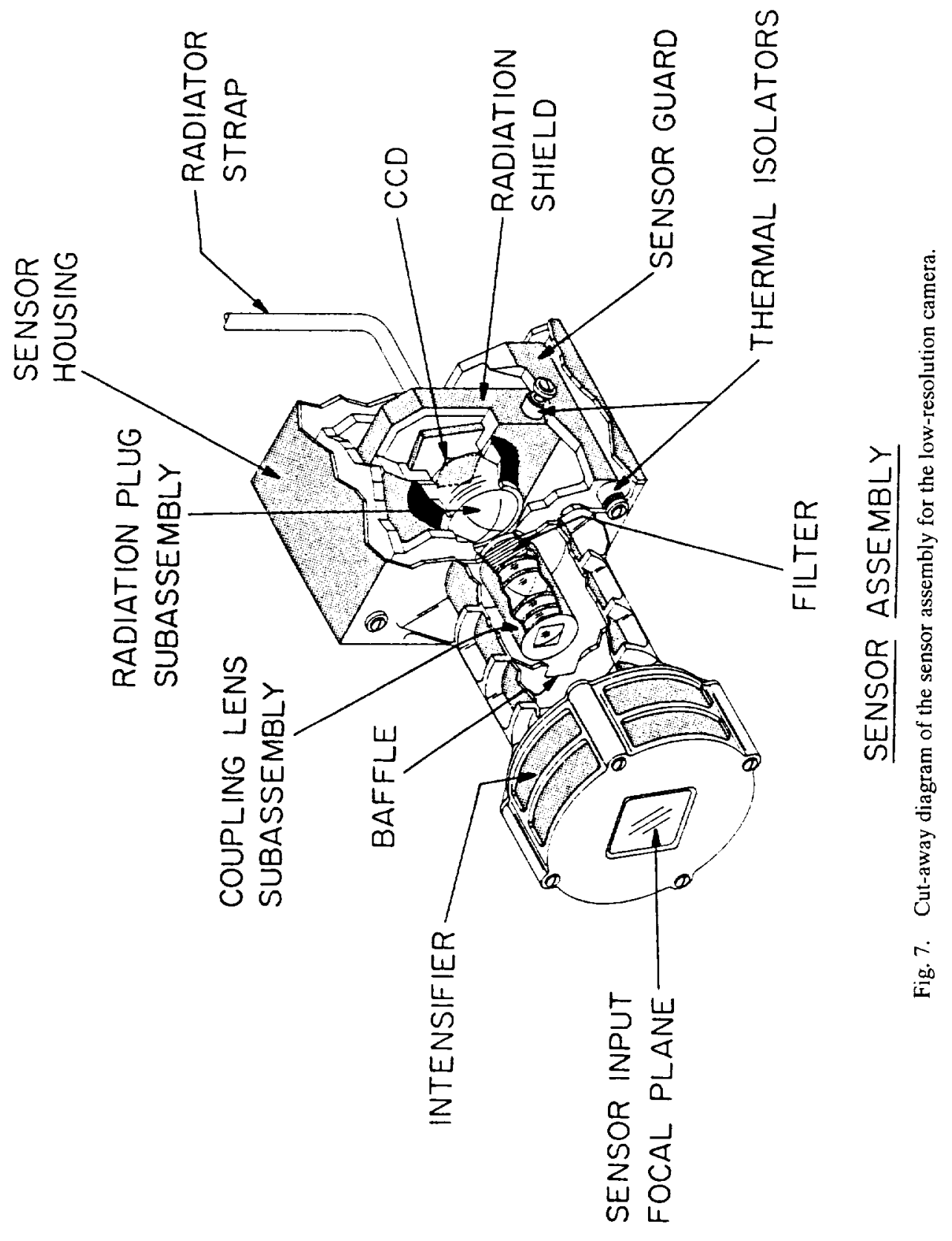


An important concern for the $\mathrm{CCD}$ is radiation degradation due to the fluxes of high-energy, penetrating protons in the inner radiation zone. Consequently, the sensor assembly was designed with a tantulum radiation shield that surrounds the entire solid angle around the CCD with the exception of the optical path. Along the optical path the radiation plug provides an equivalent minimum shielding of $5 \mathrm{gm} \mathrm{cm}^{-2}$, sufficient to stop protons with $E \leq 60 \mathrm{MeV}$. Calculations by Dyson (1991) as based upon the method of Janesick et al. (1991) indicate that the worstcase charge transfer efficiency (CTE) for a single pixel of the CCD is 0.99991 for the 300-kRad total radiation dose during a nominal three-year mission. The CTE is the measure of the performance in transferring charge from one pixel to the next pixel in the process of shifting the pixel charge packet out for A/D conversion. Thus for the pixel in row 512 and column $512,(0.99991)^{1024}=0.91$ of the charge that had accumulated in this pixel during image integration is received by the amplifier.

In order to further minimize the effects of radiation damage by energetic ions the CCD is passively cooled to a temperature of about $-90^{\circ} \mathrm{C}$ by thermal coupling to a radiator through the radiator strap (see Figure 7). The $\mathrm{CCD}$ is connected to electronics and thermally isolated as much as feasible with the use of thirteen 76- $\mu \mathrm{m}$ diameter constantan wires. At this low temperature the time constant for electron captivation in the radiation-induced traps increases to times exceeding that for image readout. That is, the electron remains in the trap and is not picked up in the signal charge packet.

\section{Electronics}

An overview of the VIS electronics is given in Figure 8. The instrument is interfaced with the spacecraft telemetry and command systems with two redundant Remote Interface Units (RIUs). The power interface is served by two independently switched and fused 28-V spacecraft power buses. Each of these power buses feeds an isolated, current-limited DC/DC converter in the instrument. In addition to these main power lines there are two separately switched, pulse power lines. The pulse power is used to drive the motors and instrument heaters. A further nonredundant power line is used for instrument survival heaters in the event that the main power lines are inactive during a spacecraft load-shedding sequence. There are six 8085 microprocessors in the instrument data and control systems. Four of these microprocessors are devoted to image compression. The details for the data compression are described in the following section. The other two microprocessors, CPU A and CPU B, are redundant and either processor can be selected for control of the instrument by ground command. Each of these processors is equipped with 48 kbytes of memory and an additional 64 kbytes of extended memory. This control processor formats data, receives commands, and controls operation of the sensors, field stop and power subsystems. These activities include bi-axial mirror control for 
mosaicing and pointing, monitoring despun platform motion, generation of antenna blanking signals for the sensors, and processing of ancillary data. The CCD video signals from the sensors are digitized by one of the two 12-bit analog-to-digital converters (ADCs). These 12-bit words are quasi-logarithmically compressed to 8 bits. An 8-kbyte data window in the memory of the control processor is remapped into the memory space of one of the compression processors. This 8-kbyte block is configured as two-port RAM. The camera interface ( $I / F)$ electronics include the serial interfaces for sensor control. Each of the three cameras shown in Figure 8 is equipped with a complete set of electronics, i.e., amplifiers, gate array, low-voltage power supply, and intensifier high-voltage supply.

The electronics are configured to significantly decrease the probability of singlepoint failure. The cameras are operated independently of each other. The instrument is normally powered by one of the two main power buses and supplies. A powerswitching network allows recovery of instrument regulated voltages in the case of failure of one or more of these power lines. The two control processors and their buses are redundant. The failure of a compression processor results in a decrease in frame rate.

\section{Data Compression}

For effective use of the telemetry rate allocated to the VIS a hybrid data compression algorithm is implemented. From the analysis of images from DE 1 it is known that the general class of images to be acquired can be characterized by an entropy (information) content of about 4 bits per 8-bit pixel. An improvement of the corresponding maximum compression ratio of $2: 1$ for completely lossless channel encoding is desired. Thus a hybrid Mean Residual Vector Quantization (MRVQ) code is employed. This code is supplemented with combined run-length and Huffman algorithms. This data compression scheme was developed under the direction of Richard Harris and Paul Israelsen of Utah State University. A Discrete Cosine Transform (DCT) was developed in parallel but was considered slightly inferior and required more onboard hardware.

Vector Quantization (VQ) is an extension of a scalar conversion. For VQ a finite group of samples denoted as a vector is compared to a list of predetermined vectors (the code book) in order to find the closest matching set. Data compression is achieved by sending the binary code (address) for the selected vector. For MRVQ a vector mean is subtracted from each sample in the vector to create a residual vector that is encoded by the VQ process. The mean and the vector selected from the code book are then telemetered. This lossy MRVQ compression is combined with a lossless Huffman coding of the error residual. In this hybrid system the error residual, which is the difference between the original and regenerated MRVQ image, is encoded using a combined variable-length Huffman and run-length algo-

rithm. In order to regulate the telemetry rate the errors in the residuals below a set 


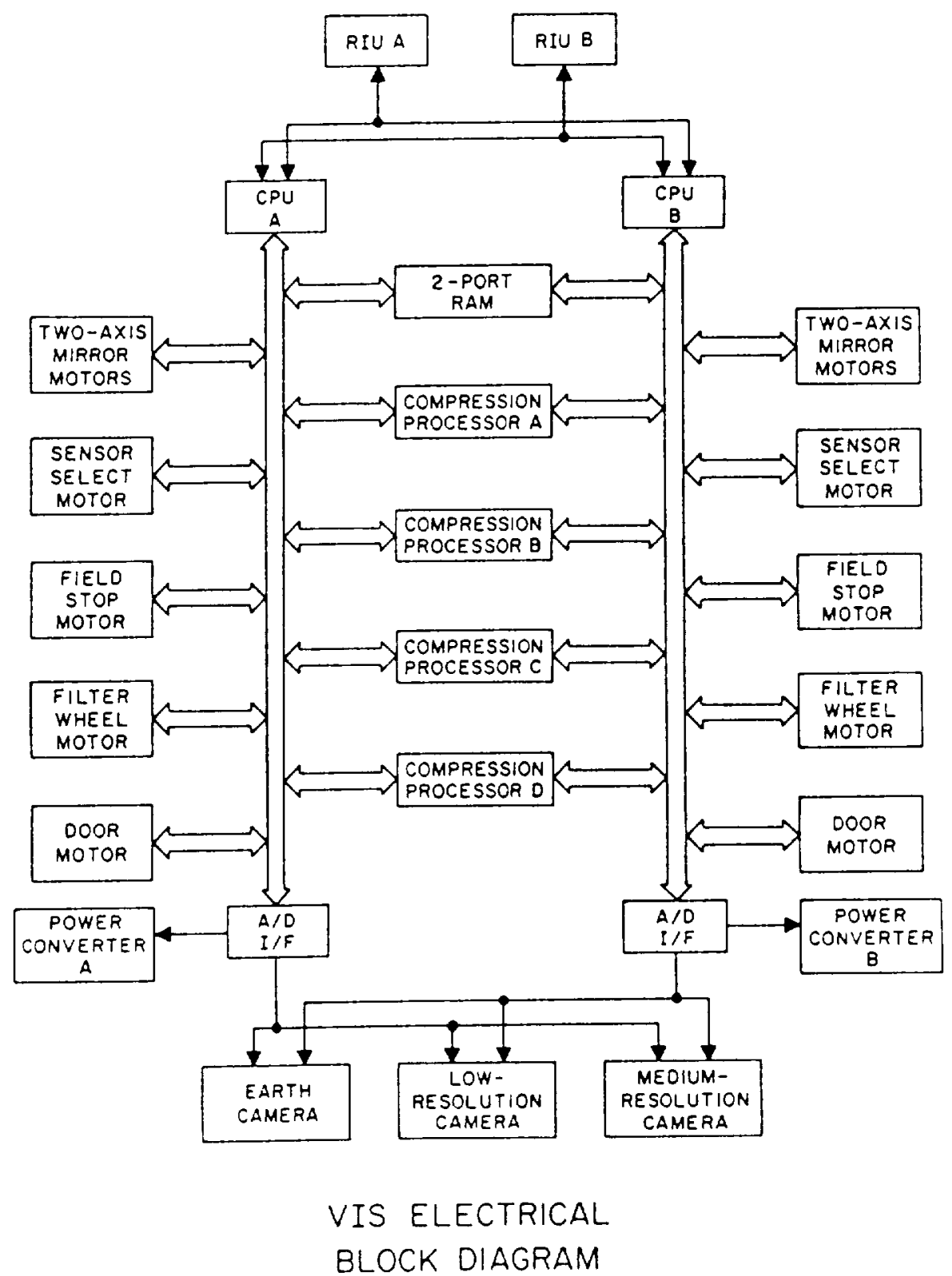

Fig. 8. Block diagram of the electronics for the VIS.

number of counts (threshold) are truncated to zero values. The resulting run-lengths of truncated zero-error-valued pixels and the nonzero Huffman-encoded residuals are telemetered. 
A reduced-resolution mean image is generated with $4 \times 4$-pixel blocks and eight-bit means. The residual vector is then encoded with the use of a 256 -vector code book and a $16 \times 16$ tree search. An error image is achieved by subtraction of this encoded MRVQ image from the original image. After the error residual is computed all such errors less than a threshold of $\pm n$ counts are set to zero. This residual is then processed using variable-length Huffman code words for individual errors or run-lengths of zero errors. For a compression ratio of $4: 1$ then 16 bits per vector (16 pixels) are allocated for encoding the residual error image. Thus the compression coding must be aggressive in order to minimize the effects on image quality.

The compression algorithm has been characterized with the use of images from DE-1 and DMSP. The DE-1 images are similar to those expected from the Earth camera. The DMSP images are anticipated to be similar to those acquired with the VIS cameras for visible wavelengths. The performance of the compression algorithm is summarized in Table V. Examination of an extensive series of compressed images finds that sharply bounded features such as thin auroral arcs are degraded significantly for compression factors $\gtrsim 5$. It may be necessary to tailor the code books and thresholds for each camera. The need for such adjustments is to be evaluated with post-launch images. The capability exists to telemeter both uncompressed and compressed images for these evaluations. It should be noted that a fixed threshold yields a variable data rate with fixed error statistics. This method has been chosen in lieu of adaptive thresholds that vary from image to image and yield a constant telemetry rate at the expense of a varying encoding error.

The data compression technique requires four 8085 microprocessors that execute in parallel. These processors utilize a multiplier accumulator chip (MAC) to implement the VQ search. The MAC is driven by three channels of a DMA controller: one channel to fetch the residual, the second to fetch the vector for comparison, and the third to write the results of the least-mean-square computation into memory. An eight-bit adder is used to subtract the mean from the vectors as they are fetched from memory. The compression processors are each equipped with 32 kbytes of memory for code and code books and three banks of 32 kbytes for image storage and output buffers.

\section{Performance}

The angular resolution of the three cameras is limited by the MTF of the image intensifiers. A typical plot of the MTF is shown in Figure 9. The dimensions of the image that is focused on the photocathode of the intensifier is $18.4 \times 18.4 \mathrm{~mm}$. For an array of $256 \times 256$ pixels the corresponding resolution is 7 line pairs $\mathrm{mm}^{-1}$. The MTF is 0.45 and adequate for auroral imaging. For an array of $512 \times 512$ pixels, or 14 line pairs $\mathrm{mm}^{-1}$, the MTF decreases to 0.15 . For this reason the CCD output is summed into $2 \times 2$-pixel blocks. This CCD pixel block is referred throughout 
TABLE $V$

Performance of image compression algorithms

\begin{tabular}{lcl}
\hline Truncation, counts pixel $^{-1}$ & R.m.s. error, counts pixel & Compression factor \\
\hline \multicolumn{3}{c}{ DE-1 images } \\
\pm 1 & $0.51 \pm 0.03$ & $2.3 \pm 0.5$ \\
\pm 2 & $0.85 \pm 0.10$ & $2.9 \pm 0.7$ \\
\pm 3 & $1.15 \pm 0.18$ & $3.5 \pm 0.7$ \\
\pm 4 & $1.43 \pm 0.33$ & $4.1 \pm 0.7$ \\
\pm 5 & $1.62 \pm 0.34$ & $5.5 \pm 0.8$ \\
& & \\
& DMSP images & \\
\pm 1 & $0.63 \pm 0.02$ & $3.2 \pm 0.7$ \\
\pm 2 & $1.00 \pm 0.05$ & $4.5 \pm 1.2$ \\
\pm 3 & $1.21 \pm 0.13$ & $5.7 \pm 1.6$ \\
\pm 4 & $1.32 \pm 0.22$ & $6.5+1.5 /-1.6$ \\
& $1.37 \pm 0.27$ & $7.1+0.9 /-1.6$ \\
\hline 5
\end{tabular}

TABLE VI

Pixej-to-pixel dimension $(256 \times 256$-pixel format $)$

\begin{tabular}{lccc}
\hline $\begin{array}{l}\text { Distance to } \\
\text { source, } R_{e}\end{array}$ & $\begin{array}{l}\text { Low-resolution } \\
\text { camera }(\mathrm{km})\end{array}$ & $\begin{array}{l}\text { Medium-resolution } \\
\text { camera }(\mathbf{k m})\end{array}$ & $\begin{array}{l}\text { Earth camera } \\
(\mathrm{km})\end{array}$ \\
\hline 1 & 2.6 & 1.3 & 8.7 \\
3 & 7.8 & 3.9 & 26.1 \\
5 & 13.0 & 6.5 & 43.5 \\
7 & 18.2 & 9.1 & 60.9 \\
9 & 23.4 & 11.7 & 78.2 \\
\hline
\end{tabular}

this paper as a pixel, although it is possible to telemeter the entire $512 \times 512$-pixel array. The angular resolution of the pixel blocks is suitable for auroral imaging. The pixel dimensions as a function of source distance for the three cameras are tabulated in Table VI.

The instrument sensitivity $S$ is defined as the charge accumulation rate in a CCD pixel per unit brightness of the emission, in units of electrons kilorayleigh ${ }^{-1}$ pixel $^{-1} \mathrm{~s}^{-1}$. The $2 \times 2$-pixel blocks are used for the calculation of sensitivity. The overall gain of the intensifier is sufficient to record individual MCP events, or avalanches, in the CCD. As an example, the sensitivity of the low-resolution camera at $557.7 \mathrm{~nm}$ is computed. The rate of intensifier avalanches, or counts, is determined by the 
MODULATION TRANSFER FUNCTION (MTF) FOR IMAGE INTENSIFIER

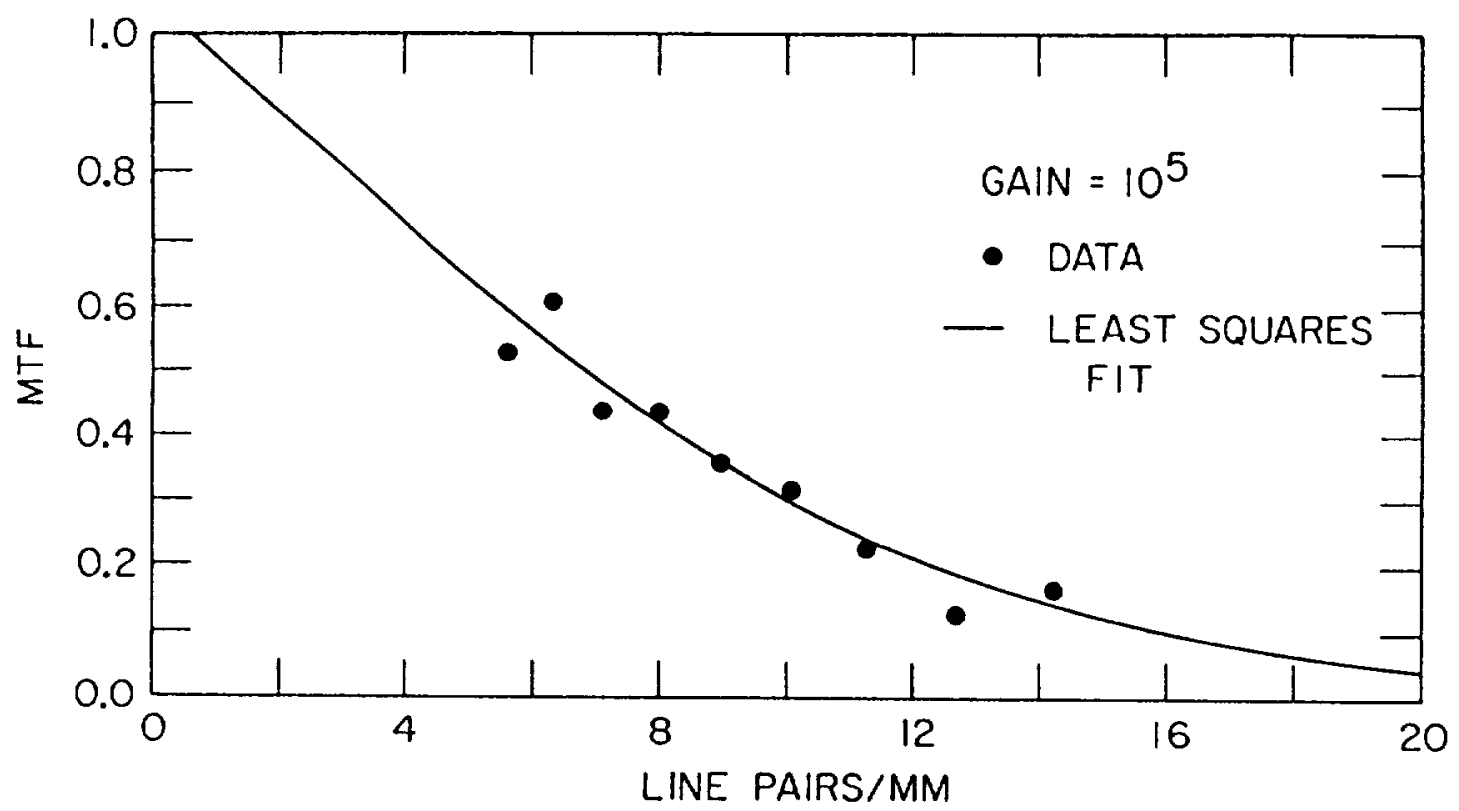

Fig. 9. Modulation transfer function for the image intensifiers.

area of the entrance pupil $A=3.14 \mathrm{~cm}^{2}$, the pixel solid angle $\Omega=1.7 \times 10^{-7} \mathrm{sr}$, reflectivity of 6 mirrors $=r^{6}$ where $r=0.90$, transmission of the passband filter $t_{f}=0.38$, transmission of the intensifier window $t_{w}=0.96$, quantum efficiency of the photocathode $q(\lambda)=0.10$, and efficiency of the MCP to initiate an avalanche $c=0.5$. Thus for a 1 -kiloRayleigh (kR) aurora, the count rate $R$ of the intensifier is

$$
R=A \Omega r^{6} t_{f} t_{w} q c\left(10^{9} / 4 \pi\right)=0.41 \text { counts } \mathrm{kR}^{-1} \mathrm{pixel}^{-1} \mathrm{~s}^{-1}
$$

The integration time for accumulation of one count in a pixel is $2.5 \mathrm{~s}$ for a $1-\mathrm{kR}$ aurora at $557.7 \mathrm{~nm}$. The light gain of the image intensifier is set by ground command to provide 100 electrons in the $2 \times 2$-pixel block of the CCD for each avalanche in the MCP. Thus the sensitivity $S$ of the low-resolution camera at $557.7 \mathrm{~nm}$ is 41 electrons $\mathrm{kR}^{-1}$ pixel $^{-1} \mathrm{~s}^{-1}$. The CCD can be operated in inverted and non-inverted modes. For fully inverted operation the full well is about $3 \times$ $10^{5}$ electrons pixel ${ }^{-1}$. The dynamic range is then approximately 3000 . The auroral intensities corresponding to a single count for several wavelengths and integration times are presented in Table VII for the low-resolution camera. A similar summary of the brightnesses for filling the CCD well in the inverted mode is given in 
TABLE VII

Threshold brightness, kR, corresponding to one count pixel ${ }^{-1}$ for low-resolution camera

\begin{tabular}{llllll}
\hline $\begin{array}{l}\text { Integration time } \\
(s)\end{array}$ & 391.4 & 557.7 & 630.0 & 656.3 & $732.0 \mathrm{~nm}$ \\
\hline 12 & 0.13 & 0.20 & 0.25 & 0.25 & 1.07 \\
24 & 0.07 & 0.10 & 0.13 & 0.13 & 0.53 \\
48 & 0.03 & 0.05 & 0.06 & 0.06 & 0.26 \\
\hline
\end{tabular}

TABLE VIII

Brightness, $k R$, corresponding to $\mathrm{CCD}$ full well for low-resolution camera

\begin{tabular}{lrrrrr}
\hline $\begin{array}{l}\text { Integration time } \\
(\mathrm{s})\end{array}$ & 391.4 & 557.7 & 630.0 & 656.3 & $732.0 \mathrm{~nm}$ \\
\hline 12 & & & & & \\
24 & 390 & 600 & 750 & 750 & 3210 \\
48 & 195 & 300 & 375 & 375 & 1605 \\
& 98 & 150 & 188 & 188 & 803 \\
\hline
\end{tabular}

Table VIII. The onset of nonlinear responses of the intensifier occurs only for the most intense aurora, $\gtrsim 200 \mathrm{kR}$, with saturation at about $600 \mathrm{kR}$. For comparison the threshold brightness for one count in a pixel for the low-resolution camera during an integration time of $12 \mathrm{~s}$ is similar for that for a pixel in a DE-1 image acquired over a time interval of $720 \mathrm{~s}$. The intensifier is operated at temperatures in the range -15 to $0{ }^{\circ} \mathrm{C}$. The background contributions at these temperatures that are due to photocathode thermionic emissions are $\lesssim 100 R$. The rate of thermionic charge accumulation in the $\mathrm{CCD}$ at $-90^{\circ} \mathrm{C}$ for inverted operation corresponds to an equivalent auroral brightness $\lesssim 1 R$. The primary source of background will be due to penetrating energetic particle fluxes when the spacecraft passes through the inner radiation zone.

There are twelve filters on the filter wheel that is shared by the low-resolution and medium-resolution cameras. These twelve filters are listed in Table IX. The wavelength at peak transmission is $\lambda_{p}, \Delta \lambda$ is the full width at half maximum for the passband, $t_{f}$ is the filter transmission at $\lambda_{p}$ and $5{ }^{\circ} \mathrm{C}$, and $S$ is the sensitivity at $\lambda_{p}$ in units of CCD electrons $\mathrm{kR}^{-1}$ pixel $^{-1} \mathrm{~s}^{-1} . S$ is given in the table for the low-resolution camera. For the moderate-resolution camera these values for $S$ must be multiplied by 4 .

The Earth camera is equipped with a single broad-band filter with full width at half-maximum transmission of $25 \mathrm{~nm}$. The passband is 124 to $149 \mathrm{~nm}$. The field- 
TABLE IX

Passband filters for the low- and medium-resolution cameras

\begin{tabular}{|c|c|c|c|c|c|c|}
\hline Filter & $\begin{array}{l}\lambda_{p} \\
\mathrm{~nm}^{\mathrm{a}}\end{array}$ & $\begin{array}{l}\Delta \lambda \\
\mathrm{nm}\end{array}$ & $t_{f}$ & $\begin{array}{l}S \\
e \mathrm{kR}^{-1} \text { pixel }^{-1} \mathrm{~s}^{-1}\end{array}$ & Source & Function \\
\hline 1 & 360.1 & 2.94 & $3.9 \times 10^{-5}$ & $7.4 \times 10^{-3}$ & surface albedo & $\begin{array}{l}\text { background for } \\
\text { ozone measurements }\end{array}$ \\
\hline 2 & 317.3 & 3.22 & $4.8 \times 10^{-5}$ & $7.3 \times 10^{-3}$ & $\mathrm{O}_{3}$ & Total ozone \\
\hline 3 & 396.4 & 2.17 & 0.34 & 73 & continuum & 391.4 background \\
\hline 4 & 531.0 & 1.13 & 0.47 & 59 & continuum & 557.7 background \\
\hline 5 & 391.4 & 2.02 & 0.29 & 63 & $\mathrm{~N}_{2}^{+} 1 \mathrm{~N}(0-0)$ & Aurora \\
\hline 6 & 557.7 & 1.02 & 0.38 & 41 & $01^{1} D-{ }^{1} S$ & Aurora \\
\hline 7 & 630.0 & 1.08 & 0.48 & 33 & $0{ }^{3} P-1 D$ & Aurora \\
\hline 8 & 732.0 & 1.10 & 0.36 & 7.8 & $\mathrm{O} \mathrm{II}^{2} D-{ }^{2} P$ & Aurora \\
\hline 9 & 626.4 & 1.09 & 0.46 & 32 & continuum & 630.0 background \\
\hline 10 & 656.3 & 1.14 & 0.40 & 33 & $\mathrm{H} \alpha$ & Aurora (proton) \\
\hline 11 & 589.0 & 1.00 & 0.52 & 47 & $\mathrm{NaI}^{2} S-{ }^{2} P$ & $\begin{array}{l}\text { atmospheric } \\
\text { nightglow }\end{array}$ \\
\hline 12 & 308.5 & 5.60 & 0.12 & 17 & $\mathrm{OH}$ & $\begin{array}{l}\text { comets, } \\
\text { atmosphere }\end{array}$ \\
\hline
\end{tabular}

a Passbands include the decrease of $\sim 0.1 \mathrm{~nm}$ for off-normal incidence on the filters.

of-view is $20^{\circ} \times 20^{\circ}$. In the $256 \times 256$-pixel format the solid angle corresponding to a pixel is $1.9 \times 10^{-6} \mathrm{sr}$. The count rate $R$ is 0.6 counts $\mathrm{kR}^{-1}$ pixel $^{-1} \mathrm{~s}^{-1}$. For a $12-\mathrm{s}$ accumulation period the single-count threshold corresponds to $140 R$. The sensitivity $S$ for $100 \mathrm{CCD}$ electrons/MCP avalanche is 60 electrons $\mathrm{kR}^{-1} \mathrm{pixel}^{-1} \mathrm{~s}^{-1}$. For operation of the CCD in the fully inverted mode the well depth is again about $3 \times 10^{5}$ electrons. For a 12 -s accumulation period the well is filled by a brightness of $420 \mathrm{kR}$. For comparison, the solid angle corresponding to a DE-1 pixel is $2.0 \times 10^{-5} \mathrm{sr}$, about 10 times larger than that of the Earth camera, with a single-count threshold of $\sim 300 R$ pixel $^{-1}$ during an accumulation time of $720 \mathrm{~s}$.

\section{Inflight Operations}

The inflight operation of the VIS cameras can be described by considerations of the DE-1 image shown at the upper left-hand side of Plate 2. This image is taken at the visible wavelength $557.7 \mathrm{~nm}$ on 2 January, 1982. The nightside aurora is shown along with the position of the terminator. This terminator is defined as a line at $8^{\circ}$ to the nightside of and parallel to the terminator at Earth's surface. The responses due to bright emissions from sunlit Earth are suppressed by reverse voltage bias 
of the sensor photocathode as triggered by the protective circuitry monitoring the pulse rate output. At an exemplary radial distance of the spacecraft position of $8.4 R_{e}$ the apparent angular size of Earth is $13.7^{\circ}$. The field-of-view of the VIS low-resolution camera is also shown by the superposed square on the DE-1 image. The low-resolution camera provides 65500 pixels within this field-of-view. For comparison the number of pixels in a DE-1 image as taken from this altitude and within the field-of-view of the low-resolution camera would be 576 pixels.

The field-of-view of the low-resolution camera is sufficient to provide monitoring of the nightside aurora in a single frame. An anticipated series of images of an auroral substorm as returned from this camera is shown in the bottom four panels of Plate 2. These anticipated images are taken from an actual sequence of images from DE-1. The frame repetition period for DE-1 is $720 \mathrm{~s}$ and is to be compared with that from the low-resolution camera, $12 \mathrm{~s}$. If the medium-resolution camera is used to obtain an image within the solid angle shown in Plate 2 then a four-frame mosaic must be taken. The corresponding number of pixels in the mosaic is 262000 pixels and the mosaic repetition period is $48 \mathrm{~s}$.

A closer examination of the image in the upper left-hand side of Plate 2 reveals that the frame from the low-resolution camera is not centered on the direction to Earth's center, i.e., along the nadir direction. The axis of the platform rotation, or clock angle, is directed normal to the orbital plane. Thus the platform rotation can allow the cameras' axis to be directed to any position along a line centered and running vertical in the DE-1 image. The bi-axial mirror motion within the VIS instrumentation allows for angular positioning of the two cameras for visible wavelengths at any position within a $20^{\circ} \times 20^{\circ}$ field-of-view. This large fieldof-view not only allows mosaicing of the entire planet at radial distances beyond $5.8 R_{e}$, but also the monitoring of apparent stellar positions for analysis of the attitude stability of the spacecraft despun platform. This stability can be monitored at high time resolution, if required, by telemetering 'postage stamp' images of the star's position. It is also obvious that the apparent position of the auroral oval changes as Earth rotates. Compensation for this apparent motion during an orbit is also accomplished with the bi-axial mirror. As the local time of the orbital plane changes during the course of a year then the viewing geometry with respect to the fields-of-view also changes. Thus the appropriate field stop must be activated to exclude sunlit Earth from being viewed by the secondary optics during certain periods. All of these functions are performed by the instrument's control processor. For each orbit the orbital parameters are uplinked, along with the desired mosaicing, field stop positions, camera type, filters, and platform clock angles. Each orbit is considered to be an observational campaign.

The instrument processor performs two further special functions. This processor provides the signals to the sensor electronics for shuttering the photocathodes when the long antennas mounted on the rotating spacecraft body are within the cameras' fields-of-view. Secondly the processor examines the Earth camera images to ensure that the location of the limb is being correctly identified from the onboard 


\section{GLOBAL AURORAL IMAGE \\ FROM DYNAMICS EXPLORER 1}

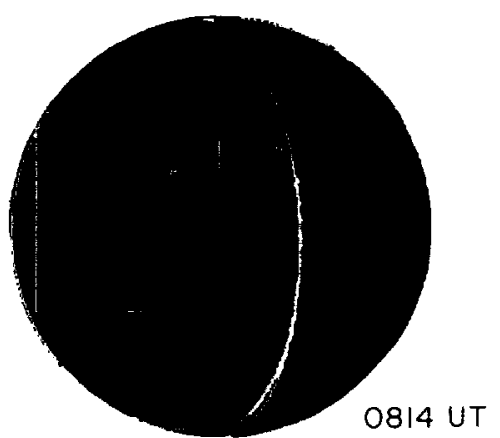

2 JANUARY 1982

\section{ANTICIPATED IMAGE FROM \\ THE VIS LOW-RESOLUTION \\ CAMERA}

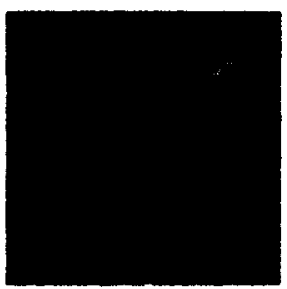

$R=8.4 R_{E}$

ANTICIPATED SUBSTORM SEQUENCE

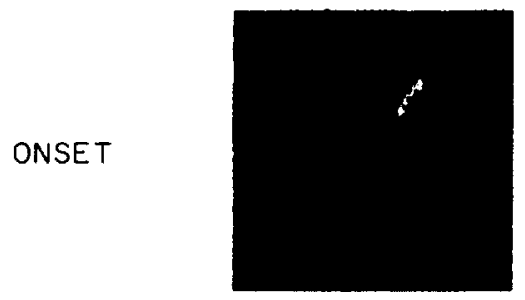

0802 UT

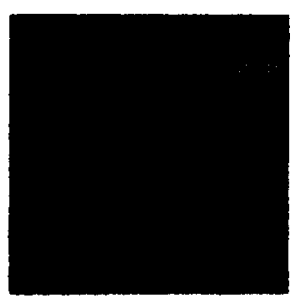

O826 UT

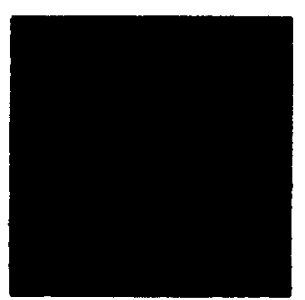

O814 UT

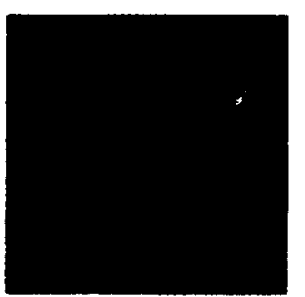

0838 UT
EARLY

EXPANSION

EARLY

RECOVERY

Plate 2. Images of atomic oxygen emissions at $557.7 \mathrm{~nm}$ from Earth's aurora as taken with one of the imaging photometers on board Dynamics Explorer 1. Anticipated images from the low-resolution camera of the VIS on the Polar spacecraft at a radial distance of $8.4 R_{e}$ are shown by the corresponding square fields-of-view.

computations based upon the uplinked orbit, orientation of the spacecraft spin axis, and platform clock angle. The field-of-view of the Earth camera, $20^{\circ} \times 20^{\circ}$, is fixed with respect to the despun platform and coincides with the full solid angle range of the cameras for visible wavelengths. Images from the Earth camera can be telemetered every $12 \mathrm{~s}$. The number of pixels is 65500 , and 5900 of these 
pixels lie within the low-resolution camera frame shown in Plate 2. At a given time the image from only one of the three cameras can be processed by the instrument subsystems. The instrument processor is responsible for formatting the data, receiving and distributing the command time line, and coordinating the activities of the four compression processors. It is anticipated that the code books for image compression will be optimized during the first month or two of inflight operations.

The key parameter (KP) data to be supplied to the Central Data Handling Facility (CDHF) for initial correlative studies with other ISTP observations consist of one image of the nightside aurora every $5 \mathrm{~min}$. The images are to be taken with the low-resolution camera for visible wavelengths at $557.7 \mathrm{~nm}$. Anticipated examples are shown in Plate 2 . The images are to be targeted at an area of frequent substorm onset, $67^{\circ}$ magnetic latitude and 2300 magnetic local time.

\section{Summary}

The Visible Imaging System (VIS) for the Polar spacecraft is presented. Global images of the nighttime aurora at visible wavelengths are to be gained over time periods that allow continual recording of all phases of a single auroral substorm. The selected wavelengths can be used to map the electron energy influxes into the auroral and polar ionospheres, and to determine the precipitation patterns of protons from the plasma sheet and its near-Earth extension, the ring current. The scientific objectives range from provision of a reference system for simultaneous insitu observations of fields and particles with magnetospheric spacecraft to studies of the global responses of the auroras as the solar wind fluctuates. Substantial improvements in the overall capabilities of the VIS relative to previously flown imagers are gained from a combination of a high-altitude polar orbit, a despun platform for staring at the auroras, improved optics and sensors, and a generous telemetry rate that is augmented with image compression.

\section{Acknowledgements}

The authors express their deep appreciation to the following University of Iowa personnel for their substantial contributions in implementing the Visible Imaging System: K. L. Ackerson, R. L. Brechwald, S. M. Cash, T. L. Clausen, L. M. Henry, E. T. Howard, E. Kruse, H. R. Peltz, P. S. Reilly, and R. M. Wenman. In addition important support and services were also provided by J. Hrastar, J. Galloway, K. O. Sizemore, M. H. Acuña, V. B. Patel, and D. Crosby of the NASA/Goddard Space Flight Center. This research was supported in part at The University of Iowa by the National Aeronautics and Space Administration under Contract NAS5-30316. 


\section{References}

Akasofu, S.-I. and Roederer, M.: 1984, Planetary Space Sci. 32, 111.

Anger, C. D., Fancott, T., McNally, J., and Kerr, H. S.: 1973, Appl. Opt. 12, 1753.

Anger, C. D., Sawchuk, W., and Shepherd, G. G.: 1974, in B. M. McCormac (ed.), 'Polar Cap Optical Aurora Seen from ISIS-2', D. Reidel Publ Co., Dordrecht, Holland, pp. 357-366.

Anger, C. D., Babey, S. K., Broadfoot, A. L., Brown, R. G., Cogger, L. L., Gattinger, R., Haslett, J. W., King, R. A., McEwen, D. J., Murphree, J. S., Richardson, E. H., Sandel, B. R., Smith, K., and Jones, A. V.: 1987, Geophys, Res. Letters 14, 387.

Coroniti, F. V. and Kennel, C. F.: 1972, J. Geophys. Res. 77, 3361.

Dyson, R. L.: 1991, 'Assessment of Energetic Proton Damage to the Visible Imaging System CCD for the POLAR Spacecraft', University of lowa Internal Report, lowa City.

Elphic, R. C., Craven, J. D., Frank, L. A., and Sugiura, M.: 1988, Phys. Scripta 37, 443.

Frank, L. A.: 1988, in T. E. Moore and J. H. Waite, Jr. (eds.), 'Dynamics of the Near-Earth MagnetotailRecent Observations', Magnetosphere/lonosphere Plasma Models, Geophys. Monogr. Ser., Vol. 44, AGU, Washington, D.C., pp. 261-276.

Frank, L. A. and Craven, J. D.: 1988, Rev. Geophys. 26, 249.

Frank, L. A. and Sigwarth, J. B.: 1993, Rev. Geophys. 31, 1.

Frank, L. A., Craven, J. D., Ackerson, K. L., English, M. R., Eather, R. H., and Carovillano, R. L.: 1981, Space Sci. Instr. 5, 369.

Frank, L. A., Craven, J. D., Gurnett, D. A., Shawhan, S. D., Weimer, D. R., Burch, J. L., Winningham, J. D., Chappell, C. R., Waite, J. H., Heelis, R. A., Maynard, N. C., Sugiura, M., Peterson, W. K., and Shelley, E. G.: 1986, J. Geophys. Res. 91, 3177.

Hallam, K. L., Howell, B. J., and Wilson, M. E.: 1983, in A. Boksenberg and D. L. Crawford (eds.), 'An All-Reflective Wide-Angle Flat-Field Telescope for Space', Instrumentation in Astronomy $V$, Proceedings of SPIE 445, London, England, pp. 295-300.

Janesick, J., Soli, G., Elliot, T., and Colins, S.: 1991, in M. M. Blouke (ed.), 'The Effects of Proton Damage on Charge Coupled Devices', Charge-Coupled Devices and Solid State Optical Sensors II, SPIE/SPSE Electronic Imaging Science and Technology Conference, Proceedings of SPIE, Vol. 1447, San Jose, California, pp. 87-108.

Kaneda, E. and Yamamoto, T.: 1991, in J. R. Kan, T. A. Potemra, S. Kokubun, and T. lijima (eds.), 'Auroral Substorms by UV-Imager on Akebono', Magnetospheric Substorms, AGU Geophys. Monogr. Ser., Vol. 64, pp. 235-240.

Kaneda, E., Takagi, M., and Niwa, N.: 1977, 'Vacuum Aurora Television Camera', Proceedings of the Twelfth International Symposium on Space Technology and Science, Tokyo, Japan, pp. 233-238.

Lyons, L. R.: 1985, J. Geophys. Res. 90, 1561.

Mendillo, M., Baumgardner, J., and Flynn, B.: 1991, Geophys. Res. Letters 18, 2097.

Meng, C.-I. and Huffman, R. E.: 1984, Geophys. Res. Letters 11, 315.

Meng, C.-I. and Makita, K.: 1986, in Y. Kamide and J. A. Slavin (eds.), 'Dynamic Variations of the Polar Cap', Solar Wind-Magnetosphere Coupling, Proceedings 126, Terra Scientific, Tokyo, Japan, pp. 605-631.

Meng, C.-1., Huffman, R. E., Del Greco, F., and Eastes, R.: 1987, EOS 68(16), 396, AGU.

Murphree, J. S., Cogger, L. L., Anger, C. D., Wallis, D. D., and Shepherd, G. G.: 1987, Geophys. Res. Letters 14, 403.

Rees, M. H., Lummerzheim, D., Roble, R. G., Winningham, J. D., Craven, J. D., and Frank, L. A.: 1988, J. Geophys. Res. 93, 12,841.

Rogers, E. H., Nelson, D. F., and Savage, R. C.: 1974, Science 183, 951.

Shepherd, G. G., Fancott, T., McNally, J., and Kerr, H. S.: 1973, Appl. Opt. 12, 1767.

Strickland, D. J., Jasperse, J. R., and Whalen, J. A.: 1983, J. Geophys. Res. 88, 8051. 